\title{
Qui est le secrétaire du tribunal arbitral ?
}

\author{
Who is the Secretary to the Arbitral Tribunal ?
}

\section{Hakem Heyeti Sekreteri Kimdir ?}

\section{Berk Hasan 0zdem ${ }^{1}$ 이}

\begin{abstract}
Résumé
Le secrétaire du tribunal arbitral est une personne qui collabore et contribue à la mission du tribunal en effectuant les tâches qui lui sont confiées par les arbitres. Bien que les secrétaires soient engagés pour les avantages qu'ils procurent, le recours à des secrétaires d'arbitrage peut également comporter des risques pouvant entraîner des contestations ou nuire au bon déroulement des procédures. Après avoir discuté des avantages et des inconvénients de cette mission, le présent document examinera les points de vue contradictoires sur les activités autorisées des secrétaires, en mettant l'accent sur les divergences entre les règles des différentes institutions arbitrales ainsi que sur les décisions de justice pertinentes. Enfin, il proposera des solutions pour minimiser les risques que les parties peuvent rencontrer.
\end{abstract}

\section{Mots-clés}

Arbitrage, Sécretaire, Intuitu Personae, Mandat, Prise de décision

\begin{abstract}
The secretary to the arbitral tribunal/arbitral secretary is a person who works with the tribunal for the purpose of contributing to the process by carrying out the tasks entrusted to him/her by the arbitrators. While the secretaries are appointed for the advantages they provide, practice of using arbitral secretaries may also entail risks that may provoke challenges or affect the smooth running of the proceedings. After discussing the benefits and not-so-bright sides of such a practice, this paper examines the contradictory views on the permissible scope of secretaries' activities by focusing on the discrepancies amongst the regulations of different arbitral institutions as well as on the relevant court decisions. Finally, it offers solutions for minimizing the risks that the parties may encounter.
\end{abstract}

\section{Keywords}

Arbitration, Secretary, Intuitu personae, Mandate, Decision making

\section{Öz}

Hakem heyeti sekreteri/hakem sekreteri, hakemler tarafından onlara verilen görevleri yerine getirerek tahkim yargılaması sürecine katkıda bulunmak amacıyla çalışan kişilerdir. Sekreterler tahkim yargılamasına sağlayacakları katkı için tayin edilmelerine rağmen, uygulamada sekreter kullanımı red taleplerine yol açabilecek veya sürecin düzgün işleyişini etkileyecek riskleri de beraberinde getirebilmektedir. Bu makalede, sekreter kullanımının yararları ve avantajlı olmayabilecek yönleri tartışıldıktan sonra çeşitli tahkim kurumlarının düzenlemelerindeki farklılıklara ve ilgili mahkeme kararlarına odaklanılarak sekreterlerin faaliyetlerinin kapsamına ilişkin çelişen görüşler incelenmektedir. Son olarak, karşılaşabileceği riskleri en aza indirmeleri için taraflara çözümler sunulmaktadır.

\section{Anahtar Kelimeler}

Tahkim, Sekreter, Intuitu personae, Yetki, Karar verme

\footnotetext{
1 Corresponding Author: Berk Hasan Ozdem (LLB), Galatasaray University, Faculty of Law, Istanbul, Turkey.

E-mail: berkhasanozdem@gmail.com ORCID: 0000-0002-0327-6489

To cite this article: Ozdem BH, “Qui est le secrétaire du tribunal arbitral?” (2019) 68 Annales de la Faculté de Droit d’Istanbul 85. https://doi.org/10.26650/annales.2019.68.0015
} 


\section{Extended Summary}

Where the identity of an arbitrator is a subjectively essential element of the arbitration agreement, in other words, where the parties choose their arbitrator in view of the specific person, the mission of the arbitrator becomes one that should not be delegated to someone else. However, the "intuitu personae" character of the mission entrusted to the arbitrator does not mean that arbitrators cannot take any assistance. Particularly in large and complex international commercial arbitrations, massive resources (such as voluminous documentary evidence or memoranda) as well as the organization and the arrangement of the proceedings may involve the sole or the presiding arbitrator in an overwhelming amount of work. In view of such a workload, in some cases it may be desirable for the arbitrators to entrust some activities to another person for assistance.

The secretary to the arbitral tribunal/arbitral secretary is a person who works with the tribunal for the purpose of contributing to the process by carrying out the tasks entrusted to him/her by the arbitrators. Particularly in large commercial arbitrations, it is a common practice for the arbitrators to appoint a secretary, generally a young lawyer, to act as a link between the parties and the tribunal as well as to secure administrative arrangements. The practice of using arbitral secretaries is generally not statutorily regulated. While some arbitration laws explicitly provide for such a possibility most jurisdictions remain silent in this regard but do not prohibit the appointment of a secretary. Furthermore, uncertanities remain as to the question whether an arbitral tribunal may appoint an arbitral secretary in a manner contrary to the consent of the parties.

When entrusted with tasks such as drafting administrative and organizational procedural orders, reviewing submissions and managing documentary evidence, the appointment of an arbitral secretary may provide great advantages to the arbitral tribunal and the parties since it can provide efficiency by leaving the arbitral tribunal with more time to concentrate on the substantive matters. Furthermore, the use of a secretary may lower the overall fees, particularly in cases where the arbitrators are remunerated on an hourly basis. Undoubtedly, it also provides an excellent opportunity for young practitionners to observe the conduct of the arbitral proceedings from the perspective of the secretary.

However, there is no rose without thorns. Despite its potential benefits, the issue of arbitral secretaries have been highly controversial across the international arbitration community. Authors mention that the use of arbitral secretaries entails risks such as ex parte communications or breaches of confidentiality which may lead to situations that could affect the swiftness of the procedure, or other perceived virtues of arbitration should they are not handled properly. Such concerns particularly make sense in cases 
where secretaries are appointed without any formal appointment process, or, in some circumstances, without any disclosure to the parties.

In addition to these risks, many scholars have been voicing concerns in recent years that through some duties they undertake, secretaries may be inappropriately doing work that a tribunal should be doing itself; and thus, violate the intuitu personae mission of arbitrators by going beyond their position as assistants and becoming decisionmakers. Contradictory views exist on the appropriate scope of secretaries' activities. While there are authors who are of the opinion that the tribunal's responsibilities include carrying out all substantive tasks, no matter adjudicative or non-adjudicative, as part of its own personal mandate, for others, even if the delegation is related to a substantive task it should be permissible as long as it does not influence the tribunal's decision-making process. In addition to the differences between the regulations of arbitral institutions, there is a paucity of case law regarding the appropriate role of the tribunal secretaries and even when the issue was considered, courts have been quite reluctant to explain the permissible functions that would not affect the decisionmaking process.

This author believes that in view of all the benefits that a secretary may provide and of all the arbitrators out there eager to fulfill their duties in the most responsible way, using arbitral secretaries should not be a practice to stay away from. However, in order to overcome, or at least to minimize, the risks entailed to such a practice, the informed consent of the parties to the appointment, the transparency about the functions of the secretary and the diligence of the arbitral tribunal from the beginning to the end of the proceedings become crucial. 


\section{Qui est le secrétaire du tribunal arbitral ?}

\section{Introduction}

En tant que « condition fondamentale de la confiance qui nourrit et vivifie l'institution tout entière $»^{1}$, le principe de base de l'arbitrage est la possibilité pour les parties de choisir les arbitres impliqués dans le règlement de leur différends. ${ }^{2}$ Les parties peuvent énoncer des qualifications et prérequis spécifiques que leurs arbitres devraient avoir (telles que l'expérience ou l'expertise dans un domaine particulier, ou la capacité de parler une langue particulière) ${ }^{3}$ et sont libres de nommer l'arbitre de leur choix. ${ }^{4}$ Lorsque l'identité d'un arbitre est un élément subjectif essentiel de la convention d'arbitrage ${ }^{5}$, en d'autres termes quand les parties choisissent un arbitre du fait de sa personne ${ }^{6}$, la mission de cet arbitre est dite sujette à l' « intuitu personae $»^{7}$ Pour cette raison, « l'arbitre doit accomplir lui-même sa mission, sans pouvoir la déléguer à un tiers $» .^{8}$

Cependant, même si la mission juridictionnelle confiée à l'arbitre est éminemment personnelle, cela ne signifie pas que les arbitres ne peuvent pas être assistés. ${ }^{9}$ Dans le

1 « La liberté du choix des arbitres - que certains centres d'arbitrage cherchent à tort à limiter- est une condition fondamentale de la confiance qui nourrit et vivifie l'institution tout entière » Pierre Lalive, 'Le choix de l'arbitre' dans Mélanges Jacques Robert, Libertés, (Montchrestien 1998) 353, 363.

2 Michal Malacka, 'Party Autonomy in the Procedure of Appointing Arbitrators' (2017) 17(2) International and Comparative Law Review 93, 95-96.

3 Margaret L. Moses, The Principles and Practice of International Commercial Arbitration (Cambridge University Press 2018) 43.

4 Joint Report of the International Commercial Disputes Committee and the Committee on Arbitration of the New York City Bar Association, 'Secretaries to International Arbitral Tribunals' (2006) 17 American Review of International Arbitration 575, 586.

5 Gabrielle Kaufmann-Kohler et Antonio Rigozzi, International Arbitration: Law and Practice in Switzerland (3 ${ }^{\mathrm{e}}$, Oxford University Press 2015) 158-159; Jean-François Poudret et Sébastien Besson, Comparative Law of International Arbitration (Sweet \& Maxwell 2007) $245 \mathrm{ff}$.

6 Michael Polkinghorne et Charles Rosenberg, 'The Role of the Tribunal Secretary in International Arbitration: A Call for a Uniform Standard' (2014) 8 Dispute Resolution International 107, 107-108; İnci Biçkin, 'Hakem Sözleşmesi ve Hakem Yargılaması' (2006) 67 Türkiye Barolar Birliği Dergisi 380, 382.

7 Pierre Lalive, 'Mission et Démission des Arbitres Internationaux' dans Marcelo Kohen, Robert Kolb et Djacoba Liva Tehindrazanarivelo (eds) Perspectives of International Law in the 21st Century / Perspectives du Droit International au 21e Siecle: Liber Amicorum Professor Christian Dominica in Honour of His 80th Birthday (Bilinguale, Brill-Nijhoff 2011) 269, 277; Guy Keutgen et Georges-Albert Dal (avec la collaboration de Marc Dal et Gautier Matray), L'arbitrage en droit belge et international ( $3^{\mathrm{e}}$, Bruylant 2015) para 264; «Il est axiomatique de dire que la mission d'un arbitre est "intuitu personae" ». Constantine Partasides, 'The Fourth Arbitrator? The Role of Secretaries to Tribunals in International Arbitration' (2002) 18 Arbitration International 147, 147, en citant Frédéric Eisemann, 'Déontologie de L'Arbitre Commercial International' (1969) 4 Revue de I'Arbitrage 217, 229; Voir aussi, Elie Kleiman 'Arbitre, Intuitu Personae' dans Laurent Lévy et Yves Derains (eds) Liber Amicorum en l'honneur de Serge Lazareff (Pedone 2011) 361; Concernant la relation contractuelle entre les parties de l'arbitrage et les arbitres en droit turc, voir, Sera Reyhani Yüksel, 'Tahkim Tarafları ve Hakem Arasında Yapılan Hakemlik Sözleşmesinin Maddi Hukuk Açısından Değerlendirilmesi’ (2016) 22 Marmara Üniversitesi Hukuk Fakültesi Hukuk Araştırmaları Dergisi: Özel Sayı Prof. Dr. Cevdet Yavuz’a Armağan 2436.

8 « La mission juridictionnelle confiée à l'arbitre est éminemment personnelle, et le contrat d'arbitre est conclu intuitu personae. Cela implique que l'arbitre doit accomplir lui-même sa mission, sans la déléguer à un tiers, fût-il un confrère travaillant dans le même cabinet que lui s'il est avocat ». Tribunal fédéral, 4A_709/2014, Arrêt du 21 mai 2015, Ire Cour de droit civil 3.2.2.; Thomas Clay, L'arbitre (Dalloz 2001) n. 422, 632, 785 et 895; Gary Born, International Commercial Arbitration (2 ${ }^{\circ}$, Kluwer Law International 2014) 2043; Lalive (n 7) 274; Francisco Blavi et Gonzalo Vial, 'The Tribunal Secretary in International Arbitrations' (2017) 30 New York International Law Review 1, 4; James U. Menz et Anya George, 'How Much Assistance Is Permissible? A Note on the Swiss Supreme Court's Decision on Arbitral Secretaries and Consultants' (2016) 33 Journal of International Arbitration 311, 313; Voir aussi, Reyhani Yüksel (n 7) 2441.

9 Kaufmann-Kohler et Rigozzi (n 5) 235. 
cas particulier d'arbitrages internationaux de grande envergure, il n'est pas rare que les parties, lorsqu'elles luttent pour leur droit, déploient des ressources considérables (telles que de nombreuses preuves documentaires ${ }^{10}$ ou des mémoires volumineux ${ }^{11}$ ) ce qui peut être une tâche accablante pour des tribunaux seulement composés d'une ou trois personnes ayant moins de vingt-quatre heures par jour à consacrer à un cas. ${ }^{12}$ Par ailleurs l'organisation d'une telle procédure peut signifier pour le président ou l'arbitre unique un travail administratif. ${ }^{13}$ Par exemple, le tribunal arbitral peut être amené à prendre des dispositions spécifiques pour organiser des réunions entre les parties et demander la présence d'interprètes lors des audiences, afin de garantir le bon déroulement de l'arbitrage. ${ }^{14}$ Lorsque l'affaire n'est pas administrée par une institution arbitrale ou que son implication ne comprend pas la fourniture d'un soutien administratif, ${ }^{15}$ ces missions retombent généralement dans les mains du tribunal luimême, plutôt que dans celle des parties. ${ }^{16}$ Par conséquent, et dans certains cas, il peut être souhaitable de confier certaines activités à une autre personne pour les aider. ${ }^{17}$

\section{Le Secretaire Du Tribunal Arbitral}

Le secrétaire du tribunal arbitral ${ }^{18}$ est une personne qui collabore et contribue au processus du tribunal en effectuant les missions qui lui sont confiées par les

10 Constantine Partasides et autres, 'Arbitral Secretaries' dans Conseil International pour l'Arbitrage Commercial, Young ICCA Guide on Arbitral Secretaries (The ICCA Reports No.1) (International Council for Commercial Arbitration 2014) 23, 27; Alexandre-Yacine Souleye, 'Fourth chair: the controversial role of arbitral tribunal secretaries' (Young ICCA Blog 16 Février 2017) <http://www.youngicca-blog.com/fourth-chair-the-controversial-role-of-arbitral-tribunal-secretaries/> consulté le 10 septembre 2019.

11 Emmanuel Gaillard et John Savage (eds), Fouchard, Gaillard, Goldman on International Commercial Arbitration (Kluwer Law International 1999) 683.

12 Zachary Douglas, 'The Secretary to the Arbitral Tribunal' dans Bernhard Berger et Michael E. Schneider (eds) Inside the Black Box: How Arbitral Tribunals Operate and Reach Their Decisions (ASA Special Series No. 42) (Juris 2014) 87, 88.

13 Alan Redfern et autres, Law and Practice of International Arbitration (4e, Sweet \& Maxwell 1999), 224.

14 ibid.

15 Voir, Commission des Nations Unies Pour le Droit Commercial International 'Draft Notes on Organizing Arbitral Proceedings: report of the Secretary-General (A/CN.9/423)' dans Yearbook of the United Nations Commission on International Trade Law Vol. XXVII (A/CN.9/SER.AI) (United Nations 1996) 45, 50.

16 «Il peut aussi être considéré comme acceptable de laisser certaines missions aux parties, ou à l'une seulement des parties $»$ ibid 50 .

17 « Cette interdiction de déléguer la tâche d'arbitrer à autrui n'exclut pas nécessairement le recours à l'assistance de tiers » Tribunal fédéral (n 8) 3.2.2.; Bernhard Berger et Franz Kellerhals, International and Domestic Arbitration in Switzerland $\left(3^{e}\right.$, Beck/Hart 2015) n. 1007; « [...] celle-ci implique souvent une documentation considérable, que bien des arbitres n'ont guère les moyens de maîtriser seuls, sans assistance » Lalive (n 7) 270, Pour Professeur Lalive, c'est pourquoi il y a « certaines "horror stories" qui circulent, oralement biensûr, entre les initiés, et qui décrivent tel praticien comme ayant démontré en audience qu'il n'avait pas eu le temps d'assimiler ou d'étudier les faits du dossier ! ».

18 En ce qui concerne la terminologie du présent article, l'auteur voudrait mentionner que les termes « secrétaire », " secrétaire du tribunal arbitral », « secrétaire d'arbitrage » et « assistant » sont utilisés indifféremment. Voir aussi, Sofia Andersson, 'A Fourth Arbitrator or an Administrative Secretary? A Study on the Appointment and Authority of Arbitral Secretaries in Swedish Arbitral Proceedings' (Mémoire de maîtrise en droit de l'arbitrage, Université d'Uppsala, 2015) 8-10; Hong-Lin Yu et Masood Ahmed, 'Keeping the Invisible Hand under Control? -Arbitrator's Mandate and Assisting Third Parties' (2016) 19(2) Vindobona Journal of International Commercial Law and Arbitration 213, 221; Pour les allégations de la Russie dans le processus d'annulation des sentences de Yukos concernant la différence entre un " secrétaire d'arbitrage » et un « assistant d'arbitrage » voir, Writ of Summons (28 janvier 2015), 181ff <https://www.italaw.com/sites/default/ files/case-documents/italaw4158_0.pdf> consulté le 10 septembre 2019 ; Concernant le fond de Yukos, voir, Bağdagül Kaya Caner, 'Enerji Şartı Anlaşmasının Muvakkaten Uygulanması ve Anlaşmadan Çekilme Hakkında Bir Değerlendirme' (2014)3 Ankara Barosu Dergisi 315, 328-329; voir aussi générallement, Nihal Üral, 'Avrupa Enerji Şartı Antlaşmasının Onaylanmamış Olmasının Hakem Mahkemesinin Yetkisi Üzerindeki Etkileri: Yukos Davası' 2009 8(1) İstanbul Kültür Üniversitesi Hukuk Fakültesi Dergisi 71. 
arbitres. Ils sont couramment assignés par les institutions arbitrales à quelques litiges administrés par elles. ${ }^{19}$ Lorsque l'institution d'arbitrage ne nomme pas de secrétaire, ou dans le cadre d'une procédure d'arbitrage ad hoc, certains arbitres engagent fréquemment les mêmes personnes. ${ }^{20}$ Bien qu'une étude menée par White \& Case et par l'Université Queen Mary de Londres révèle que des secrétaires n'étaient nommés que dans 35\% des affaires, ${ }^{21}$ un autre sondage réalisée en 2012 dans le cadre du congrès du Conseil International pour l'Arbitrage Commercial (ICCA) à Singapour indique que 95\% des participants approuvent l'utilisation du secrétaire. ${ }^{22}$ Dans le cas particulier des arbitrages commerciaux internationaux importants et complexes, les arbitres ont pour habitude de nommer un secrétaire chargé de faire le lien entre les parties et le tribunal et de mettre en œuvre des procédures administratives que le tribunal arbitral aurait autrement été amené à prendre lui-même. ${ }^{23}$

Les secrétaires peuvent ou non faire partie de l'institution arbitrale qui va juger d'un litige. ${ }^{24}$ Dans l'enquête menée par l'ICCA, seulement $14,3 \%$ des personnes interrogées ont déclaré qu'une liste fournie par une institution d'arbitrage était le lieu idéal pour trouver un secrétaire potentiel, tandis que $69,4 \%$ ont indiqué qu'elles nommeraient un employé du cabinet d'avocats du président du tribunal ou de l'arbitre unique. ${ }^{25}$ En ce qui concerne le niveau et le type d'expérience qu'un secrétaire devrait avoir, la pratique montre que, en fonction de leurs besoins, les choix des parties diffèrent selon les profils : étudiants en droit, ${ }^{26}$ jeunes professionnels ${ }^{27}$ ou même

19 Commission des Nations Unies Pour le Droit Commercial International (n 15) 50.

20 ibid.

21 Selon l'enquête internationale sur l'arbitrage menée en 2012 par White \& Case et l'Université Queen Mary de Londres, auprès de 710 personnes interrogées, les secrétaires sont plus souvent nommés dans les arbitrages concernant de civil law (46\%) par rapport aux arbitrages du common law (24\%). D'un point de vue régional, le recours aux secrétaires des tribunaux est plus fréquent dans les arbitrages d'Amérique latine (62\%), tandis qu'il est moins fréquent dans les arbitrages d'Amérique du Nord (23\%) et d'Asie (26\%). White \& Case et Queen Mary University of London School of International Arbitration, 2012 International Arbitration Survey: Current and Preferred Practices in the Arbitral Process, $11<\mathrm{http}$ :/www.arbitration.qmul.ac.uk/media/arbitration/docs/2012_International_Arbitration_Survey.pdf $>$ consulté le 10 septembre 2019.

22 Conseil International pour l'Arbitrage Commercial, Young ICCA Guide on Arbitral Secretaries (The ICCA Reports No.1) (International Council for Commercial Arbitration 2014) 55.

23 Redfern et autres (n 13) 224; Berger et Kellerhals (n 17) 356; Une autre enquête qui concerne spécialement la pratique en Afrique afin de comprendre l'expérience des sondés dans l'arbitrage entre les années 2012 et 2017, indique que parmi les 191 réponses reçues « [e]n matière d'arbitrage interne, environ $2 \%$ des répondants sont intervenus comme secrétaire de tribunal arbitral dans 11 à 15 arbitrages, tandis qu'en matière d'arbitrage international, on compte entre six et dix références seulement pour environ $2 \%$ des répondants. Aucun répondant n'est intervenu en qualité de secrétaire du tribunal dans au moins 15 différends en matière d'arbitrage international au cours de la période de référence. » SOAS University of London et Broderick Bozimo Company, Enquête SOAS sur l'Arbitrage en Afrique Arbitrage Interne et International: Perspectives par les praticiens africains de l'arbitrage 2018, 19 <https://eprints.soas.ac.uk/26110/1/Enquête\%20SOAS\%20sur\%20 $1 \% 27$ arbitrage \%20en\%20Afrique-Fr.pdf> consulté le 10 septembre 2019.

24 Bridie Mcasey, 'International Arbitral Institutions' dans Freya Baetens (ed), Legitimacy of Unseen Actors in International Adjudication (Cambridge University Press 2019) 51, 67.

25 Conseil International pour l'Arbitrage Commercial (n 22) 57.

26 ibid.

27 Voir, Keutgen et Dal (n 7) para 264; Partasides (n 7) 147. 
arbitres réputés. ${ }^{28}$ Cependant, l'enquête de 1'ICCA révèle que les avocats débutants étaient entre autres les plus privilégiés pour faire fonction de secrétaires. ${ }^{29}$

\section{A. Les réglementations nationales concernant les secrétaires d'arbitrage}

Le sujet des secrétaires d'arbitrage n'est généralement pas réglementé par la loi. ${ }^{30}$ La plupart des juridictions restent silencieuses à cet égard mais n'interdisent pas la nomination d'un secrétaire. ${ }^{31}$ Alors que le Concordat Suisse de $1969^{32}$ et la loi espagnole sur l'arbitrage de $1988^{33}$ qui reconnaissent expressément la possibilité pour les tribunaux arbitraux de nommer des secrétaires sont abrogés par des lois qui ne contiennent aucune disposition à cet égard, la loi brésilienne prévoit toujours une telle possibilité. ${ }^{34}$ La Cour d'appel de Paris a admis qu'un tribunal arbitral pouvait nommer un secrétaire et que celui-ci pouvait assister aux audiences sans porter atteinte à l'égalité des parties. ${ }^{35}$ De même le Tribunal Fédérale suisse a jugé qu'est "généralement admis que la possibilité, offerte par l'art. 365 al. 1 CPC (RS 272) au tribunal arbitral, de désigner un secrétaire dans un arbitrage national vaut aussi en matière d'arbitrage international, quand bien même le chapitre 12 de la LDIP ne la mentionne pas. $\gg{ }^{36}$ Les lois de certains pays comme le Chili ${ }^{37}$ ou l'Argentine ${ }^{38}$ rendent la désignation d'un secrétaire obligatoire.

\section{B. Les réglementations nationales concernant les secrétaires d'arbitrage}

Il convient de mentionner que des incertitudes subsistent quant à la question de savoir si un tribunal arbitral peut nommer un secrétaire arbitral d'une manière contraire

28 Voir, Eric A. Schwartz, 'The Rights and Duties of ICC Arbitrators' dans Jean-François Bourque (ed) Special Status of the Arbitrator, ICC International Court of Arbitration Bulletin: 1995 Special Supplement (ICC Publishing 1995) 67, 86; « Il n'est pas surprenant que des praticiens talentueux aient servi ou continuent à servir de secrétaires à d'éminents tribunaux arbitraux. Pour en nommer quelques uns : M. Martin Valasek dans HYV v Russian Federation (Yves Fortier, Stephen Schwebel, Charles Poncet); M. Samuel Wordsworth QC dans Methanex v USA (William Rowley, Warren Christopher, V.V. Veeder); Dr. Romesh Weeramantry dans McKenzie v Vietnam (Neil Kaplan, Campbell McLachlan, John Gotanda), et bien d'autres. » Olga Boltenko, 'Stop Ignoring the Elephant in the Room!' (Kluwer Arbitration Blog 9 juin 2014) <arbitrationblog. kluwerarbitration.com/2014/06/09/stop-ignoring-the-elephant-in-the-room-2/> consulté le 10 septembre 2019.

29 Selon le sondage, les interrogés recherchent des secrétaires ou assistants de bureau (\%1), des assistants juridiques ( $\% 6.1)$, des étudiants en droit (\%9.2), des avocats stagiaires (\%26.5), des jeunes avocats (\%89.8), des avocats expérimentés $(\% 26.5)$ et des jeunes arbitres $(\% 25.5)$ comme secrétaires de tribunal arbitral. Conseil International pour l'Arbitrage Commercial (n 22) 57.

30 Mauro Rubino-Sammartano, International Arbitration Law and Practice, (3 $3^{\mathrm{e}}$, Juris 2014) 537.

31 Voir, Sebastián Partida, L'arbitre international : étude de droit comparé (Master 2 Recherche de Droit européen comparé, Université Paris II Panthéon - Assas, Séjour de recherche à la Harvard Law School 2011) $48<\mathrm{https}$ ://docassas.u-paris2.fr/ nuxeo/site/esupversions/6078e606-e9d8-4733-b52a-1bdd14eb402f > consulté le 10 septembre 2019. 
à l'accord des parties. Dans une décision prise en $2015^{39}$ sur la base d'une convention d'arbitrage ad hoc dont les règles de procédure n'étaient pas déterminées par les parties, le Tribunal fédéral suisse a expliqué que la lex arbitri suisse ne stipulait pas que le consentement des parties était nécessaire pour la nomination d'un secrétaire arbitral. Il a toutefois noté que les parties pouvaient conjointement exclure une telle possibilité, que ce soit dans la convention d'arbitrage ou à un stade ultérieur :

« [...] le projet du Conseil fédéral soumettait la désignation d'un secrétaire à l'accord des parties (FF 2006 7103), mais cette condition a été abandonnée à l'instigation du Conseil des Etats pour favoriser l'autonomie organisationnelle du tribunal arbitral et éviter des retards (BO 2007 CE 641). Doit cependant être réservée la volonté commune des parties, exprimée dans la convention d'arbitrage ou dans un accord ultérieur, d'exclure la désignation d'un secrétaire. $»^{40}$

Bien que l'on puisse penser à première vue que le Tribunal fédéral a jugé que la possibilité pour les tribunaux arbitraux de nommer des secrétaires soit subordonnée à l'accord des parties, les auteurs précisent que, lorsqu'il a fait cette déclaration, le Tribunal fédéral suisse s'est référé au manuel de Tarkan Göksu' ${ }^{41}$, qui note par la suite que même si les parties déclarent conjointement qu'elles n'acceptent pas la nomination, le tribunal arbitral peut toujours retenir les services d'un secrétaire d'arbitrage, à condition que la rémunération du secrétaire ne soit pas supportée par les parties. ${ }^{42}$ En l'espèce, le Tribunal fédéral fédéral n'a pas été confronté à un tel scénario puisqu'il n'y avait pas d'opposition commune des parties à la nomination du secrétaire. ${ }^{43}$ Par conséquent, il est mentionné que la déclaration du Tribunal fédéral suisse n'est «pas assez spécifique » pour être interprétée comme ayant statuée sur la question de savoir si le tribunal arbitral peut retenir d'office l'usage d'un secrétaire d'arbitrage contre la volonté des parties et la question reste controversée dans la doctrine juridiques suisse. ${ }^{44}$ Cependant, mis à part les discussions relatives à la lex arbitri suisse, de nombreux auteurs affirment que les parties devraient être libres de choisir si leurs arbitres peuvent ou non faire appel à un secrétaire ${ }^{45}$ et que

39 Tribunal fédéral, 4A_709/2014, Arrêt du 21 mai 2015, Ire Cour de droit civil 3.2.2.

40 ibid.

41 Tarkan Göksu, Schiedsgerichtsbarkeit (Dike 2014) para 880.

42 Michael Feit et Chloé Terrapon Chassot, 'The Swiss Federal Supreme Court Provides Guidance on the Proper Use of Arbitral Secretaries and Arbitrator Consultants under the Swiss lex arbitri: Case Note on DFC 4A_709/2014 dated 21 May 2015' (2015) 33 ASA Bulletin 897, 907; Concernant la question de la rémunération les arbitrages internationaux voir Kemal Dayındarlı, ‘'̇htiyarî Tahkimde Hakem Ücretleri' dans Yaşar Karayalçın (ed), Prof. Dr. Ali Bozer'e Armağan, (Bankacılık Enstitüsü Yayınları 1998) 539.

43 ibid.

44 ibid; Jean Marguerat et Tomás Navarro Blakemore, 'Note: A. SA v. B. Sàrl, Federal Supreme Court of Switzerland, 1st Civil Law Chamber, Case No. 4A_709/2014, 21 May 2015’ 201613 Revista Brasileira de Arbitragem 199, 203-204.

45 Voir par exemple, International Commercial Disputes Committee and Committee on Arbitration of the New York City Bar Association (n 4) 591; Douglas (n 12) 88; Simon Maynard, 'Laying the fourth arbitrator to rest: re-evaluating the regulation of arbitral secretaries' (2018) 34 Arbitration International 173, 183; Benjamin Hughes, 'The Problem of Undisclosed Assistance to Arbitral Tribunals' dans Patricia Shaughnessy et Sherling Tung, The Powers and Duties of an Arbitator: Liber Amicorum Pierre A. Karrer (Kluwer Law International 2017) 161, paras 17.01-17.03; Blavi et Vial (n 8) 12; Waincymer, Procedure and Evidence in International Arbitration (Kluwer Law International 2012) 445. 
"l'autonomie des parties est en contradiction avec toute tentative de réglementer l'utilisation du secrétaire sans le consentement des parties ou en contravention avec leur autorité. $\rangle^{46}$

\section{La rose et ses épines}

\section{Les bons aspects de la nomination d'un secrétaire}

Comme toute méthode alternative de règlement des litiges, l'arbitrage international s'efforce de maximiser l'efficacité de la justice qu'il offre ; en d'autres termes, il s'efforce d'accroître la vitesse et de réduire les coûts de la procédure. ${ }^{47}$

Ce n'est pas un secret de la communauté des arbitres que de dire qu'ils sont généralement des personnes aux agendas bien remplis. ${ }^{48}$ Parallèlement à leurs mandats ad hoc - parfois plusieurs arbitrages en parallèle $e^{49}$-, les arbitres accomplissent généralement diverses tâches supplémentaires : un nombre important d'entre eux exerce dans des cabinets d'avocats ; certains d'entre eux enseignent et se sont engagés à partager leurs connaissances; et beaucoup d'autres sont des professionnels travaillant à temps plein dans des secteurs éclectiques..$^{50}$ Dans des affaires complexes en particulier, la nomination de secrétaires peut offrir de grands avantages à l'arbitrage international, dans la mesure où il permet de gagner en efficacité en laissant au tribunal arbitral plus de temps pour se concentrer sur les questions de fond. ${ }^{51}$ En utilisant judicieusement un secrétaire, le tribunal arbitral et les parties peuvent bénéficier d'une assistance pour garantir que les communications et les documents, ainsi que le déroulement procédural de l'affaire elle-même, sont gérés correctement et efficacement, permettant ainsi au tribunal de se concentrer sur l'essentiel de l'affaire et ne pas se concentrer sur l'arbre qui cache la forêt. ${ }^{52}$

46 Courtney J. Restemayer, 'Secretaries Always Get a Bad Rep: Identifying the Controversy Surrounding Administrative Secretaries, Current Guidelines, and Recommendations' (2012) 4 Yearbook on Arbitration Mediation 328, 337-338; Voir, Tracey Timlin, 'The Swiss Supreme Court on the Use of Secretaries and Consultants in the Arbitral Process' (2016) 8 Yearbook on Arbitration Mediation 268, 294.

47 Partasides (n 7) 156; Restemayer (n 46) 329; Yavuz H Alangoya, Medeni Usul Hukukumuzda Tahkimin Niteliği ve Denetlenmesi (İstanbul Üniversitesi 1973) 15; Fikret Yıldırım, 'Tahkimde İptal Davası ve İptal Davasının Amaçları Bakımından Bazı Değerlendirmeler' (2016) 22 Marmara Üniversitesi Hukuk Fakültesi Hukuk Araştırmaları Dergisi: Özel Sayı Prof. Dr. Cevdet Yavuz'a Armağan 3006, 3018; Voir généralement, Loukas A. Mistelis, 'Efficiency. What Else? Efficiency as the Emerging Defining Value of International Arbitration: between Systems theories and party autonomy' (15 avril 2019) Queen Mary School of Law Legal Studies Research Paper No. 313/2019<https://ssrn.com/abstract=3372341> consulté le 10 septembre 2019.

48 Souleye (n 10).

49 Menz et George (n 8) 318.

50 Souleye (n 10).

51 Doug Jones, 'Ethical Implications of Using Paralegals and Tribunal Secretaries' (2014) 17 Hors Serie 251, 253; 'On rappelle brièvement les avantages du secretaire, connus de tous ceux qui ont expérimenté le systeme : économie de temps et d'argent' Pierre Lalive, 'Inquiétantes dérives de l'arbitrage CCI (sur un recent "Oukase" du Secrétariat de la Cour d'Arbitrage CCI)' (1995) ASA Bulletin 634, 636.

52 James U. Menz, Miss Moneypenny vs. the Fourth Musketeer: the Role of Arbitral Secretaries (Kluwer Arbitration Blog 9 juillet 2013) <arbitrationblog.kluwerarbitration.com/2013/07/09/miss-moneypenny-vs-the-fourth-musketeer-the-role-ofarbitral-secretaries/> consulté le 10 septembre 2019; Blavi et Vial (n 8) 8; Partasides et autres (n 10) 27. 
De plus, il est mentionné que le recours aux secrétaires est une méthode largement utilisée pour réduire les coûts. ${ }^{53}$ Lorsque le secrétaire d'arbitrage est chargé de tâches telles que la rédaction d'ordonnances de procédure administratives et organisationnelles, l'examen des affaires soumises et la gestion de preuves documentaires, cela réduit le temps que les arbitres auraient passé dessus. En conséquence, la nomination d'un secrétaire d'arbitrage peut réduire les honoraires globaux du tribunal arbitral, en particulier dans les cas où les arbitres sont rémunérés à 1 'heure. ${ }^{54}$ Comme le montrent clairement les résultats de l'enquête de 2012 , le potentiel d'économie de coûts est l'une des principales raisons de recourir à un secrétaire. Parmi les sondés qui ont approuvé la pratique consistant à nommer des secrétaires, 58,8\% d'entre eux ont indiqué que les économies de coûts étaient l'une des principales raisons de la nomination d'un secrétaire et $57,7 \%$ ont indiqué que leur objectif était notamment de gagner du temps, lequel est directement lié aux coûts pour les cas où les arbitres sont rémunérés à l'heure..$^{55}$

En outre, être secrétaire d'un tribunal arbitral offre « une excellente formation et une excellente éducation pour les jeunes professionnels ${ }^{56}$. Étant donné que de nombreux cabinets ont maintenant des services dédiés à l'arbitrage, les jeunes collaborateurs ont la possibilité d'observer le déroulement d'un arbitrage du point de vue du secrétariat. ${ }^{57}$ Le professeur Pierre Lalive, réputé « être un ardent défenseur de l'utilisation des secrétaires au fil des ans ${ }^{58}$, résume les avantages d'une telle expérience :

«[...] Plus importante encore est l'occasion aussi donné aux arbitres de demain de faire leur apprentissage et à juste très juste titre que M. Shihata, Secrétaire général du CIRDI, a insisté dans son excellente conclusion au 12e Colloque CCI - AAA - CIRDI du 17 novembre, sur l'importance prioritaire de la formation des arbitres. Rien ne peut remplacer pareille formation pratique, pas même la fréquentation assidue des colloques ou un stage au Secrétariat de la Cour CCI. » ${ }^{59}$

En résumé, le recours à un secrétaire d'arbitrage présente certains avantages pour la procédure, ce qui pourrait, selon Blavi et Vial, être la raison pour laquelle $95 \%$ des

53 Arthur W. Rovine, Contemporary Issues in International Arbitration and Mediation: The Fordham Papers (Brill - Nijhoff 2010) 139.

54 Partasides et autres notent que les honoraires du tribunal arbitral et du secrétaire sont inférieurs aux honoraires de l'avocat des parties dans la plupart des procédures arbitrales, sinon toutes. Ainsi, le versement supplémentaire à un secrétaire d'arbitrage, qui s'accompagnerait généralement de frais généraux moins élevés pour le tribunal arbitral, ne devrait pas alarmer. Partasides et autres (n 10) 32; Kyriaki Karadelis, 'The Role of the Tribunal Secretary' (Global Arbitration Review 21 décembre 2011) <www.globalarbitrationreview.com/news/article/30051/the-role-tribunal-secretary/> consulté le 10 septembre 2019.

55 Conseil International pour l'Arbitrage Commercial (n 22) 15-16 et 56.

56 Jones (n 51) 253; Blavi et Vial (n 8) 8.

57 Nigel Blackaby et autres, Redfern and Hunter on International Arbitration (6 $6^{e}$, Oxford University Press 2015) 252.

58 Voir, Partasides (n 7) 148.

59 Lalive 1995 (n 51) 636; L'enquête menée par l'ICCA a révélé que près de 22\% des personnes interrogées qui approuvent l'emploi de secrétaires ont déclaré que l'un des principaux objectifs de la nomination d'un secrétaire est de « fournir à un jeune collaborateur une expérience pratique de l'arbitrage ». Conseil International pour l'Arbitrage Commercial (n 22) 56. 
personnes interrogées dans le sondage de l'ICCA ont indiqué avoir approuvé une telle pratique. ${ }^{60}$

\section{Les moins bons aspects de la nomination d'un secrétaire}

Cependant, il n'y a pas de rose sans épines. En dépit des avantages potentiels mentionnés ci-dessus, le sujet des secrétaires a été très controversé dans la communauté de l'arbitrage international. ${ }^{61}$ Les auteurs mentionnent que l'utilisation de secrétaires comporte des risques tels que des divulgations ex parte ${ }^{62}$ ou des violations de la confidentialité pouvant conduire à des situations affectant la bonne conduite de la procédure ou d'autres vertus de l'arbitrage si elles ne sont pas traitées correctement. ${ }^{63}$

Dans de nombreuses juridictions, les arbitres sont soumis à une obligation de confidentialité qui est généralement considérée comme l'un des principaux avantages de l'arbitrage et qui peut être extrêmement valorisée par les parties. ${ }^{64} \mathrm{~A}$ cet égard il est noté que « le secrétaire doit respecter le secret qui s'attache à la procédure arbitrale en général et aux pièces et renseignements échangés au cours de celle-ci en particulier $\gg{ }^{65}$. La préoccupation relative à la confidentialité est particulièrement pertinente dans les cas où les secrétaires sont nommés sans processus de nomination officiel ou, dans certaines circonstances, sans aucune information aux parties. ${ }^{66}$

60 Blavi et Vial (n 8) 9; En ce qui concerne ces avantages, voir aussi, Ole Jensen, Tribunal Secretaries in International Arbitration (Oxford University Press 2019) 51ff; Pierre Lalive, 'Un Post-Scriptum et Quelques Citations' (1996) ASA Bulletin 35, 35-43.

61 Katia Fach Gómez, Key Duties of International Investment Arbitrators: A Transnational Study of Legal and Ethical Dilemmas (Springer 2019) 198.

62 Le groupe de travail de la Young ICCA sur la nomination et l'utilisation de secrétaires d'arbitrage mentionne qu' « il est important de noter que toute communication du secrétaire faite au nom du tribunal arbitral doit clairement indiquer qu'elle est faite en tant que tel et doit obéir aux mêmes règles que celles applicables aux communications entre les parties et le tribunal arbitral, en particulier les règles relatives aux communications ex parte.' Conseil International pour l'Arbitrage Commercial Arbitration (n 22) 12.

63 Jones (n 51) 253; Blavi et Vial (n 8) 10 et 13; Restemayer (n 46) 337.

64 Blavi et Vial (n 8) 13; Gaillard et Savage (n 11) 1132; Concernant l'obligation de confidentialité dans l'arbitrage, voir généralement, Eric Loquin 'Les obligations de confidentialité dans l'arbitrage' (2006)2 Revue de l'Arbitrage 323; Philippe Cavalieros, 'La Confidentialité de l'Arbitrage' (2006)2 Les Cahiers de l'Arbitrage 56; Born (n 8) 2249-2287; Cemre Tüysüz, 'Milletlerarası Ticari Tahkimde Gizlilik Yükümlülüğü' (2017) 37(2) Public and Private International Law Bulletin 846; Ergun Özsunay, 'Tahkim Yargılamasında Mahremiyet' (2004)2 İstanbul Barosu Dergisi 89; Zeynep Derya Tarman, 'Tahkim ve Gizlilik' (2008)1 Maltepe Üniversitesi Hukuk Fakültesi Dergisi 305; Candan Yasan, 'Milletlerarası Tahkimde Gizlilik' (2011)1 Galatasaray Üniversitesi Hukuk Fakültesi Dergisi 777.

65 Keutgen et Dal (n 7) para 264; «Le secrétaire doit être lié par les mêmes devoirs de confidentialité et de secret que le tribunal arbitral ». Conseil International pour l'Arbitrage Commercial (n 22) 8; Voir, Richard M. Mosk 'Deliberations of Arbitrators' dans David Caron et autres (eds), Practising Virtue: Inside International Arbitration 486, 495; Poudret et Besson (n 5) 513; Voir aussi, Restemayer (n 46) 337.

66 Dans l'enquête de 2012, 72,4\% des interrogés étaient en faveur du consentement des parties, tandis qu'une minorité ont qualifié l'exigence de consentement en fonction de circonstances différentes. En réponse à une version différente de la question dans l'enquête de 2013, 74,7\% des personnes interrogées ont convenu qu'un tribunal arbitral ne devrait pas être autorisé à utiliser un secrétaire d'arbitrage en l'absence d'une nomination officielle et de la connaissance des parties. Conseil International pour l'Arbitrage Commercial (n 22) 58-60 (questions 8 et 11) 74-75 (questions 9,10, 11 et 12); Selon un sondage mené par Berwin Leighton Paisner, $76 \%$ des interrogés ont indiqué que le consentement des parties devrait être une condition préalable à la nomination d'un secrétaire au tribunal, Berwin Leighton Paisner, Research based report on the use of tribunal secretaries in international commercial arbitration (2015 Berwin Leighton Paisner) $3<\mathrm{https} / /$ www-staging.bclplaw.com/images/content/1/4/v2/147226/BLP-International-Arbitration-Survey-2015.pdf> consulté le 10 septembre 2019." 
Quand un tribunal demande l'assistance d'un secrétaire de cette manière, ce qui est vivement critiqué par certains auteurs ${ }^{67}$, il est dit que «l'obligation de maintenir la confidentialité devient diluée et ambiguë $\gg .{ }^{68} \mathrm{Il}$ en va de même pour les devoirs essentiels d'indépendance et d'impartialité lorsque les règles applicables les prévoient pour le secrétaire ${ }^{69}$, car le fait de nommer un secrétaire à l'insu des parties les prive de la possibilité de refuser la nomination ou de contester la sentence en se fondant sur des doutes quant à l'impartialité ou à l'indépendance du secrétaire. ${ }^{70}$

En outre, contrairement à ceux qui affirment que les parties peuvent bénéficier de la rentabilité ${ }^{11}$ du secrétaire, il est mentionné que son recours pourrait présenter un désavantage en termes de coûts pour les parties car ces coûts pourraient augmenter en tenant compte des paiements additionnels aux honoraires de 1'arbitre ${ }^{72}$. Le problème de la rémunération est encore plus important dans les cas où des secrétaires sont nommés sans que les parties en soient informées. Hughes explique le problème comme suit:

«L'un des aspects les plus troublants de l'utilisation non divulguée d'assistants au sein des tribunaux arbitraux est la question de la rémunération. Lorsque le tribunal est rémunéré sur une base ad valorem, conformément aux règles de la CCI ou de la SIAC, aucun problème particulier ne se pose. Peut-être on présume que l'assistant serait payé sur les honoraires versés à l'arbitre ou au tribunal, lesquels ne sont pas sensiblement affectés par le nombre d'heures facturées. Cependant, lorsque le tribunal est payé sur une base horaire, comment doit-on rémunérer les heures d'un assistant non divulgué ? Par exemple, dans le contexte d'un cabinet d'avocats, il semble peu probable que tout le temps consacré à un arbitrage par un avocat collaborateur soit simplement

67 «C'est donc avec étonnement que, lors d'un intéressant Colloque organisé en 2009 par la School of International Arbitration de Queen Mary College, Londres, nous avons entendu l'un des "panelists", praticien genevois connu, soutenir le caractère normal et justifié de la délégation par l'arbitre, à un collaborateur, de sa fonction de décision. Et ceci sur la base du consentement, tacite et présumé des parties en litige ! » Pierre Lalive (n 7) 277;

« L'autonomie des parties est en contradiction avec toute tentative de réglementation de l'utilisation des secrétaires sans le consentement des parties ou de leur autorité principale.» Restemayer (n 46) 337-338; Hughes (n 45) paras 17.01-17.03; Blavi et Vial (n 8) 12; Waincymer (n 45) 445.

68 Blavi et Vial (n 8) 13.

69 Par exemple, l'article 15 du Concordat suisse soumettait les secrétaires d'arbitrage aux mêmes exigences d'indépendance et d'impartialité que les arbitres. Concordat sur l'arbitrage du 27 mars 1969, art.15; Voir, Kaufmann-Kohler et Rigozzi (n 5) 312; Poudret et Besson (n 5) 513. Voir aussi concernant l'indépendance et l'impartialité des arbitres, Ziya Akınc1, Milletlerarası Tahkim (4e, Vedat Kitapçılık 2016) 158ff; Didem Kayalı, Milletlerarası Ticarî Tahkimde Hakemlerin Bağımsızlığı ve Tarafsızlığı (Seçkin 2015); Berk Hasan Özdem et Mehmet Yusuf Sert, 'Uluslararası Tahkimde Çıkar Çatışması Hakkında Uluslararası Barolar Birliği Kılavuzu'nun İncelenmesi’ (2019) 8(1) Uluslararası Ticaret ve Tahkim Hukuku Dergisi 151.

70 Voir, Hughes (n 45) para 17.02; Mcasey (n 24) 68.

71 Voir, supra (n 28).

72 International Commercial Disputes Committee and Committee on Arbitration of the New York City Bar Association (n 4) 584; Menz et George (n 8) 318; Blavi et Vial (n 8) 10; Restemayer (n 46) 330; Afin de prendre des précautions sur cette question, la Cour International d'Arbitrage note que « Toute rémunération due au Secrétaire administratif doit être prélevée sur la somme totale des fonds alloués pour les honoraires des arbitres, de façon à éviter toute majoration du coût total de l'arbitrage. En aucun cas le tribunal arbitral ne doit demander aux parties un défraiement au titre des activités du Secrétaire administratif. Toute entente sur les honoraires du Secrétaire administratif faite directement entre les parties et le tribunal arbitral est prohibée. » Chambre de Commerce Internationale Cour International d'Arbitrage, Note aux Parties et aux Tribunaux Arbitraux sur la Conduite de l'Arbitrage selon le Reglement d'Arbitrage CCI (Cour International d'Arbitrage 2019) para 191-192 <https://cms.iccwbo.org/content/uploads/sites/3/2016/11/icc-note-to-parties-and-arbitral-tribunalson-the-conduct-of-arbitration-french.pdf> consulté le 10 septembre 2019. 
éludé. L'associé désigné comme arbitre pourrait-il simplement s'approprier les heures facturées par son collaborateur? Ou est-ce que certains calculs seraient appliqués pour réduire le taux de facturation et/ou le nombre d'heures du collaborateur afin d'éviter de surcharger les parties? Comment peut-on arriver à une telle formule? Les mines antipersonnel éthiques et le potentiel de méfait sont évidents. $\gg^{73}$

\section{Le noeud de la controverse}

En complément de ces risques "le nœud de la controverse $»^{74}$ entourant les secrétaires repose sur l'influence que revêt ce poste. Ces dernières années, de nombreux universitaires ont exprimé leur inquiétude quant au fait que les secrétaires remplissent parfois certaines fonctions qu'un arbitre devrait lui-même accomplir, et donc violent la mission intuitu personae des arbitres en allant au-delà de leur rôle d'assistants et en devenant des décideurs. ${ }^{75}$ Cette inquiétude qui a engendré la création de surnoms pour les secrétaires tels que « the Fourth Arbitrator $»{ }^{76}$ « the Fourth Chair $»,{ }^{77}$ « the Fourth Musketeer $»,{ }^{78}$ « the Invisible Hand $»,{ }^{79}$ « the Unseen Actor $»,{ }^{80}$ « le Collaborateur ${ }^{81}$ et «the Elephant in the Room » ${ }^{82}$ a été soulignée dans les enquêtes 2012 et 2013 de l'ICCA par les réponses aux questions relatives aux tâches qu'un secrétaire d'arbitrage devrait être autorisé à accomplir. Les sondages indiquent que, même si les tâches administratives ont reçu un support considérable, les réponses ont commencé à changer radicalement à mesure que les tâches envisagées sont devenues plus substantielles..$^{83}$ Par exemple, 95,6\% des personnes interrogées ont voté en faveur de l'organisation de réunions et d'auditions par des secrétaires, tandis que $74,2 \%$ d'entre elles ont indiqué que les secrétaires ne devraient pas être autorisés à donner leur point de vue au tribunal arbitral. ${ }^{84}$

Alors, quelles sont les missions qui inquiètent les commentateurs ? La réponse n'est pas simple. Les arbitres de différents systèmes juridiques, et même au sein d'une même juridiction, ont des points de vue contradictoires sur la portée appropriée

\footnotetext{
73 Hughes (n 45) para 17.02.

74 Thomas Clay, 'Le secrétaire arbitral' (2005) Revue d'Arbitrage 931, 931; Restemayer (n 46) 338.

75 Polkinghorne et Rosenberg (n 6) 107; Mcasey (n 24) 67; Jones (n 51) 253; Blavi et Vial (n 8) 9; « La désignation d'un secrétaire du tribunal arbitral n'affecte pas la mission de l' arbitre, qui est intuitu personae, ni la responsabilité qui en découle. En d'autres termes, quand bien même les parties ont marqué leur accord à la désignation d' un secrétaire, les arbitres ne peuvent s' abriter derrière cette désignation pour écarter totalement ou partiellement leur éventuelle responsabilité à l'égard des parties ou du centre d'arbitrage qui les a désignés. » Keutgen et Dal (n 7) para 264.

76 Partasides (n 7) 147,

77 Souleye (n 10).

78 Menz 2013 (n 52).

79 Yu et Ahmed (n 18).

80 Mcasey (n 24) 67.

81 Pierre Lalive, 'L'Article 190 al. 2 LDIP a-t-il une utilité ?' (2010) 28 ASA Bulletin, 726, 728; Lalive (n 7) 277.

82 Boltenko (n 28).

83 Conseil International pour l'Arbitrage Commercial (n 22) 2-3.

84 ibid 3.
} 
des activités des secrétaires. ${ }^{85}$ Certains auteurs sont d'avis que la responsabilité du tribunal inclut notamment l'exécution de toutes les missions de fond, qu'elles soient ou non de nature juridictionnelle, dans le cadre de son mandat, Pour d'autres, même si la délégation est liée à une tâche de fond, elle devrait être admissible tant qu'elle n'influence pas le processus décisionnel du tribunal. ${ }^{86}$

En effet, il est clair qu'un secrétaire ne devrait pas être impliqué dans le processus de prise de décision ${ }^{87}$; cependant, la détermination des fonctions qui n'auraient aucune incidence sur le processus décisionnel du tribunal n'est pas une tâche facile, car il n'est pas si simple de déterminer où ce processus commence et se termine. ${ }^{88}$ Par exemple, bien qu'il soit incontestable pour Ashford qu'un secrétaire devrait s'acquitter des « tâches de bureau » telles que la tenue d'un registre ${ }^{89}$, Restemayer demande :

« si le secrétaire réalise les procès-verbaux des débats sans surveillance, ou peutêtre sans formation de sténographe judiciaire, comment savoir dans quelle mesure est dissimulée son opinion, son agenda, sa perception des parties? Bien qu'il ne soit pas impensable de fournir une documentation impartiale, ces rapports ne sont pas soumis à un examen par une autorité pour confirmer leur véracité. Des opinions mêmes non intentionnelles pourraient abonder dans les documents que les arbitres examineront avant de rendre la sentence. Un arbitre présent à la conférence GAR [Global Arbitration Review] a déclaré que les faits et les arguments qui se dégagent pendant la procédure sont indispensables pour identifier le raisonnement qui sous-tend une sentence. Si le compte rendu est vraiment essentiel pour le résultat, ces pratiques courantes, et souvent d'arrière-plan, garantissent un cadre. $»^{90}$

Les auteurs expriment leur préoccupation devant des fonctions telles que la recherche juridique, ${ }^{91}$ la rédaction d'un résumé de cette recherche sur un point de droit, ${ }^{92}$ la rédaction de chronologies factuelles et de mémorandums résumant les

85 Rovine (n 53) 142; « Les secrétaires remplissent une large gamme de tâches et de fonctions. Ils peuvent apporter un appui purement organisationnel, notamment effectuer des réservations de salles d'audience et de réunion et fournir ou coordonner des services administratifs. Certains tribunaux arbitraux leur confient des tâches plus fonctionnelles, notamment sous forme de recherches juridiques ou d'autres types d'assistance professionnelle, comme établir des résumés des faits ou de l'historique de la procédure arbitrale, élaborer des recueils ou des résumés de décisions de justice ou de commentaires publiés sur des questions juridiques définies par le tribunal, ou rédiger des projets de décisions de procédure. » Commission des Nations Unies Pour le Droit Commercial International, Aide-mémoire de la CNUDCI sur l'organisation des procédures arbitrales (Mars 2012) $16<\mathrm{https://www.uncitral.org/pdf/french/texts/arbitration/arb-notes/arb-notes-2016-}$ ebook-f.pdf> consulté le 10 septembre 2019.

86 Yu et Ahmed (n 18) 222-223; Partasides et autres (n 10) 27-28.

87 Rhéaume v. Société d'Investissements L'Excellence Inc. [2010] QCCA (Québec Ct. App.) 2269, para 25; Threlfall v Fanshawe (1850) 19 LJQB 334; « Le secrétaire ne peut en aucun cas prendre la place des arbitres auxquels il est interdit de déléguer la mission qui leur est confiée. » Keutgen et Dal (n 7) para 264; Âlim Taşkın, Hakem Sözleşmesi, (Turhan Kitabevi 2005) 126; Reyhani Yüksel (n 7) 2441.

88 Peter Ashford, Handbook on International Commercial Arbitration (Juris 2009) 143; Restemayer (n 46) 339; Blavi et Vial (n 8) 4.

89 Ashford (n 88) 143.

90 Restemayer (n 46) 339, citing Karadelis (n 54).

91 Commission des Nations Unies Pour le Droit Commercial International (n 15) para 27.

92 Yu et Ahmed (n 18) 224. 
observations et les éléments de preuve des parties, ${ }^{93}$ la compilation des ressources, la gestion de la documentation unique des procédures ${ }^{94}$ et, par-dessus tout, la préparation des projets de sentence ${ }^{95}$, peut causer des problèmes en raison de l'influence qu'ils peuvent avoir sur le processus décisionnel du tribunal arbitral.

\section{E. Les divergences entre les règles des différentes institutions arbitrales}

Certains auteurs affirment que le débat a été alimenté par les différentes restrictions imposées aux fonctions des secrétaires dans les notes ou directives de différentes institutions arbitrales. ${ }^{96}$ En fait, les institutions d'arbitrage commercial ont été « l'avant-garde $»^{97}$ en matière de réglementation des activités des secrétaires des tribunaux arbitraux aux côtés de la Commission des Nations-Unies pour le Droit Commercial International et du Conseil International pour l’Arbitrage Commercial.

93 Voir, Guillermo Aguilar-Alvarez, 'Foreword' dans Conseil International pour l'Arbitrage Commercial, Young ICCA Guide on Arbitral Secretaries (The ICCA Reports No.1) (International Council for Commercial Arbitration 2014) vii, vii; Ashford (n 88) 143.

94 Restemayer (n 46) 338-339; Feit et Chassot (n 42) 897.

95 En réponse à la question de savoir s'il est approprié que la tâche de rédaction de la sentence soit déléguée au secrétaire, certains auteurs déclarent que le tribunal ne devrait en aucun cas être libéré de son obligation de rédiger lui-même la sentence. Voir par exemple, « [C]omment en effet l'arbitre pourrait-il se contenter d'indiquer à son secrétaire ou "law clerk" dans quel sens il doit rédiger la sentence? Comment admettre que la forme de celle-ci serait indépendante du fond et donc laissée à l'activité du secrétaire? À l'évidence, contenu et expressions de celui-ci sont inséparables et interdépendants, et c'est finalement lors de la rédaction finale, dans le choix des mots, que l'arbitre parviendra à une relative certitude quant à la justesse, et à la justice, de sa décision. La mission de l'arbitre international "intuitu personae", ne permet donc en principe aucune dichotomie, aucune délégation de ce genre. » Lalive (n 7) 277; « [U]n arbitre devrait limiter le rôle du secrétaire dans la rédaction des sentences. » Maynard (n 45) 182; «Au cours d'un examen contradictoire, on a demandé pourquoi et on s'est demandé comment certains arbitres pouvaient traiter autant de litiges. Une solution consiste à confier la rédaction à d'autres, dans le cas du CIRDI, au Secrétariat. Il semble que les arbitres, très occupés, apprécient beaucoup, mais c'est inapproprié. » Compañia de Aguas del Aconquija SA \& Vivendi Universal SA v Argentine Republic, ICSID Case No ARB/97/3 (Annulment Proceeding), Additional Opinion of Professor Jan Hendrik Dalhuisen under Art 48(4) of the ICSID Convention, 30 juillet 2010, [8]; Selon une autre approche, tant que le tribunal fournit les orientations et que le projet est soumis à un examen minutieux des arbitres, il est inutile de limiter un secrétaire à une telle tâche. Voir par exemple, Lars Heuman, Arbitration Law of Sweden: Practice and Procedure (Juris 2003) 493; Feit et Chassot font valoir que la Cour fédérale suisse implique également cette approche dans son obiter dictum qui prévoit que « Les tâches du secrétaire juridique sont comparables à celles d'un greffier en procédure étatique: organisation des échanges d'écritures, préparation des audiences, tenue du procès-verbal, établissement des décomptes de frais, etc. Elles n'excluent pas une certaine assistance dans la rédaction de la sentence, sous le contrôle et conformément aux directives du tribunal arbitral ou, s'il n'est pas unanime, des arbitres majoritaires, ce qui suppose que le secrétaire assiste aux audiences et aux délibérations du tribunal arbitral. » Feit et Chassot (n 42) 908; Tribunal fédéral, 4A_709/2014, Arrêt du 21 mai 2015, Ire Cour de droit civil 3.2.2.; Une troisième approche suggère que les secrétaires peuvent être autorisés à rédiger des parties non substantielles des sentences, qui peuvent consister en une description de l'identité des parties et de leur conseil, de l'historique de la procédure et d'un bref résumé des faits non controversés. Voir par exemple, Partasides (n 7) 158; «Il est admis qu'un secrétaire ou un arbitre assistant est autorisé à rédiger la partie introductive d'une sentence telle que celle décrivant l'identité des parties et de leur conseil et, le cas échéant, l'historique procédural et un bref aperçu des faits non controversés. » Waincymer (n 45) 446; «Par conséquent, le secrétaire peut rédiger des ordonnances de procédure et des parties non substantielles des sentences, telles que le contexte de la procédure et les positions des parties. » Polkinghorne et Rosenberg (n 6) 126. A la lumière des résultats de différentes enquêtes, cette approche semble être préférée. White \& Case et Queen Mary University of London School of International Arbitration (n 21) 43; Conseil International pour l'Arbitrage Commercial (n 22) 15, 79; International Commercial Disputes Committee and Committee on Arbitration of the New York City Bar Association (n 4) 584-585.

96 Polkingthorne et Rosenberg ne voient aucune bonne raison pour des institutions arbitrales différentes de placer des restrictions considérables et appellent à une meilleur uniformisation des règles. Polkinghorne et Rosenberg (n 6) 107-108, 121; cf. il est peu probable qu'une norme uniforme satisfasse toutes les parties et il ne semble pas y avoir de raison de ne pas multiplier la pratique permettant aux secrétaires d'arbitrage de se voir confier des tâches différentes, en fonction des règles institutionnelles choisies par les parties. Maynard ( $\mathrm{n} 45) 183$.

97 Mcasey (n 24) 67. 
Quant aux divergences entre les activités autorisées pour un secrétaire, certaines institutions ont préféré proposer des réglementations plus libérales. Par exemple, les directives de la JAMS sur l'utilisation des greffiers et des secrétaires dans les arbitrages ${ }^{98}$ laisse aux arbitres un large pouvoir discrétionnaire quant aux fonctions du secrétaire, à condition que celui-ci ne participe pas aux délibérations ou au processus décisionnel. Cette approche est également suivie par l'article 3(1) des «Best Practices for the Appointment and Use of Arbitral Secretaries » de la Young ICCA, qui suggère que le rôle du secrétaire peut légitimement aller au-delà des tâches purement administratives placées sous la direction et la supervision appropriées du tribunal et comprend des tâches telles que la recherche de questions de droit, la recherche de questions relatives aux preuves factuelles et aux dépositions des témoins, la rédaction d'ordonnances de procédure et de documents similaires, la rédaction de chronologies et de notes résumant les exposés et les preuves des parties, la participation aux débats du tribunal et la rédaction de passages pertinents de la sentence. ${ }^{99} \mathrm{D}$ 'autres règles, cependant, telle que la note sur la conduite de l'arbitrage de la $\mathrm{CCI}^{100}$ et la Practice Note de la $\mathrm{SIAC}^{101}$ viennent limiter les devoirs du secrétaires à des matières purement administratives. ${ }^{102}$

98 Judicial Arbitration and Mediation Services, Guidelines for Use of Clerks and Tribunal Secretaries in Arbitrations $<$ https://www.jamsadr.com/files/Uploads/Documents/JAMS-International-Guidelines-for-Use-of-Clerks-and-TribunalSecretaries-in-Arbitrations.pdf $>$ consulté le 10 septembre 2019.

99 Conseil International pour l'Arbitrage Commercial (n 22) 5.

100 Cour International d'Arbitrage (n 72) paras 177-192; La rigueur de la note de la CCI à l'égard des secrétaires d'arbitrage a été mise en lumière lors de débats intéressants. En réponse à la plainte du professeur Lalive concernant l'augmentation du nombre de sentences de mauvaise qualité, Schweizer a souligné le rôle purement administratif du secrétaire fourni dans la note de la CCI.: «Une dernière remarque, et c'est surtout pour cela que je vous écris : vous avez milité ardemment, auprès de la CCI notamment, pour la reconnaissance du statut de secrétaire du tribunal arbitral, fonction à vocation préparatoire qui devait notamment permettre de former les ténors et les cadors du surlendemain. Et vous dénoncez maintenant "une augmentation des sentences de qualité médiocre, confuses ou erronées, et mal rédigées-que ce soit par l'arbitre lui-même ou (selon une mode en inquiétant progrès) par un collaborateur auquel il aurait délégué sa tâche" D'abord, selon les directives CCI, les prérogatives d'un secrétaire de tribunal arbitral s'épuisent dans le droit qu'on peut éventuellement lui reconnaître de réserver des chambres d'hôtel single ou doubles, avec ou sans hôtesse, avant l'audience, et sur place de commander au mieux les panini chips, les cafés piscines et les eaux minibulles qui agrémenteront les papotages de pauses. Ensuite, si l'on regarde les choses en face, le secrétaire, conscient du privilège que lui confère le président qui l'adoube, accomplit un travail de forme et de fond au moins aussi minutieusement qu'un arbitre débordé. Et à moindre frais. Pour moi l'art d'un bon arbitre surbooké c'est d'ordonner, de décider, de diriger, de (re)lire, de corriger, ou pour faire plus simple : de donner son avis s'il en a un. Et dans $99 \%$ des cas tout cela se passe à la satisfaction générale et dans un anonymat, pour ne pas dire camouflage, total où chacun trouve son compte (bancaire, sauf peut-être le secrétaire). Aussi ne vous laisseraije pas dire, sans me rebiffer ne serait-ce que d'un lever de sourcil, qu'une partie de la qualité médiocre des sentences suisses est due à l'appel en cause, si je puis dire, de "collaborateurs" !» Philippe Schweizer, 'Correspondance Au Sujet de L'Article 190(2) LDP: Quelques lignes en réponse à l'article du Professeur Lalive « L'article 190 al. 2 LDIP a-t-il une utilité ? ”' (2011) 29 ASA Bulletin 66, 69; Lalive 2010 (n 81) 728.

101 Singapore International Arbitration Center, Practice Note for Administered Cases - On the Appointment of Administrative Secretaries (2 février 2015) <http://www.siac.org.sg/our-rules/practice-notes/practice-note-for-administered-cases-on-theappointment-of-administrative-secretaries> consulté le 10 septembre 2019.

102 Commission des Nations Unies Pour le Droit Commercial International 2012 (n 85); London Court of International Arbitration, LCIA Notes for Arbitrators, Section $8<\mathrm{https}$ //www.lcia.org/adr-services/lcia-notes-for-arbitrators.aspx $>$ consulté le 10 septembre 2019; Hong Kong International Arbitration Center, Guidelines on the Use of a Secretary to the Arbitral Tribunal <https://www.hkiac.org/images/stories/arbitration/HKIAC\%20Guidelines\%20on\%20Use \%20 of\%20Secretary\%20to\%20Arbitral\%20Tribunal\%20-\%20Final.pdf> consulté le 10 septembre 2019; Australian Centre for International Commercial Arbitration, ACICA Guideline on the Use of Tribunal Secretaries 10-13<https:/acica. org.au/wp-content/uploads/2017/01/ACICA-Tribunal-Secretary-Guideline.pdf> consulté le 10 septembre 2019; Finland Arbitration Institute, Note on the Use of a Secretary $<$ https://arbitration.fi/wp-content/uploads/sites/22/2013/06/note-onthe-use-of-a-secretary.pdf> consulté le 10 septembre 2019. 


\section{Les décisions de justice pertinentes}

En complément des différences entre les réglementations des institutions d'arbitrage, il existe peu de jurisprudence concernant le rôle des secrétaires et, même lorsque la question a été examinée, les tribunaux sont restés assez réticents à expliquer quelles seraient les fonctions autorisées qui n'auraient aucune incidence sur le processus de résolution du litige.

Dans l'affaire Sacheri c. Robotto ${ }^{103}$ de 1989, la Cour Suprême Italienne a été confrontée à une situation dans laquelle les arbitres, qui n'avaient aucune formation juridique, ne participaient pas à la rédaction de la sentence et avaient chargé un avocat de les rédiger. Soulignant que les arbitres ne peuvent pas déléguer leur devoir de décision, la Cour Suprême Italienne a noté que

« du fait de l'incapacité déclarée des arbitres à trancher des questions autres que des problèmes techniques de construction, il revenait à déléguer à un tiers le soin de formuler la décision finale, ce que les arbitres n'étaient pas en mesure de concevoir et qu'ils ne pouvaient pas examiner de manière critique une fois qu'elle avait été rédigée $»^{104}$

La Cour a estimé que l'implication de la tierce partie constituait une violation des garanties d'une procédure régulière et a annulé la sentence contestée. Cependant, la délégation de la fonction décisionnelle était claire dans ce cas.

Dans l'affaire Sonatrach c. Statoil,${ }^{105}$ la compagnie pétrolière nationale algérienne Sonatrach avait demandé l'annulation d'une sentence de la Chambre de Commerce international d'une valeur de 536 millions de dollars américains en faveur de la compagnie pétrolière nationale norvégienne Statoil. Dans sa lettre aux parties du 17 janvier 2011, le président du tribunal avait expliqué sa volonté de nommer un secrétaire comme suit:

"Le tribunal arbitral serait heureux de compter sur l'assistance d'un secrétaire administratif. Le statut du secrétaire administratif consistera uniquement à assister le Tribunal et son président dans les tâches administratives liées à la procédure, à l'organisation des audiences et à la préparation des documents pouvant être utiles à la décision. En aucun cas le secrétaire administratif n'aura le droit de participer à la décision. $»^{106}$

Aucune objection n'a été soulevée par les parties lorsque le président a invité les parties à confirmer si elles approuvaient la nomination. Sonatrach a ensuite affirmé que le tribunal avait indûment délégué ses pouvoirs au secrétaire puisqu'il avait

103 Sacheri v. Robotto, Corte di Cassazione, 2765, 7 juin 1989 extrait de "Decision" disponible dans Albert Jan van der Berg, Yearbook Commercial Arbitration Volume XVI (International Council for Commercial Arbitration 1991) 156-157.

104 ibid, Decision para 1.

105 [2014] EWHC 875 (Comm).

106 ibid [47]. 
permis au secrétaire de produire des notes à l'attention du tribunal qui avaient ensuite été utilisées dans les délibérations. Selon Sonatrach une telle tâche ne pouvait être considérée comme administrative. Toutefois, le président du tribunal a confirmé que le secrétaire n'avait pas outrepassé ses missions, telles que décrites dans la lettre du 17 janvier 2011. Même si elle a mentionné que «les notes faisaient partie intégrante des délibérations $»{ }^{107}$, la Haute Cour de justice d'Angleterre et du pays de Galles a finalement rejeté la demande de Sonatrach.

Andersson explique que le tribunal de district de Halmstad a discuté du type de tâches régulièrement confiées aux secrétaires d'arbitrage dans une affaire ${ }^{108}$ liée à la réduction de la rémunération d'un arbitre. ${ }^{109}$ Dans cette affaire, bien que l'ampleur des tâches du secrétaire n'ait pas été réglée par un accord, celui-ci avait assisté à toutes les audiences et délibérations, mené des recherches juridiques et examiné et structuré les preuves et documents de référence qu'il avait ensuite présentés au tribunal. ${ }^{110}$ Le tribunal de district rejeta la demande de réduction de la rémunération de l'arbitre, inter alia au motif que les tâches exécutées par le secrétaire n'étaient que des tâches incombant habituellement aux secrétaires. ${ }^{111}$ Toutefois, étant donné que l'affaire concernait la rémunération de l'arbitre plutôt que la contestation ou l'invalidité de la sentence, Andersson indique qu'il est impossible de conclure si les types de tâches susmentionnés peuvent être délégués à un secrétaire en vertu de la loi suédoise sur l'arbitrage. ${ }^{112}$

Au travers d'un obiter dictum dans sa décision ${ }^{113}$ du 21 mai 2015, le Tribunal fédéral suisse a également exprimé son opinion sur le rôle du secrétaire :

«Les tâches du secrétaire juridique sont comparables à celles d'un greffier en procédure étatique: organisation des échanges d'écritures, préparation des audiences, tenue $d u$ procès-verbal, établissement des décomptes de frais, etc. Elles n'excluent pas une certaine assistance dans la rédaction de la sentence, sous le contrôle et conformément aux directives du tribunal arbitral ou, s'il n'est pas unanime, d'une majorité des arbitres, ce qui suppose que le secrétaire assiste aux audiences et aux délibérations du tribunal arbitral. Il lui est, en revanche, interdit, sauf convention contraire des parties, d'exercer des fonctions de nature judiciaire, lesquelles doivent demeurer l'apanage des seuls arbitres. $\rangle^{114}$

107 ibid [49]-[50].

108 Halmstad District Court case no. T 271-83.

109 Andersson (n 18) 55.

110 ibid.

111 ibid.

112 Andersson note également que les jugements des tribunaux de district n'ont pas une grande valeur en tant que sources du droit en Suède. ibid.

113 Tribunal fédéral, 4A_709/2014, Arrêt du 21 mai 2015, Ire Cour de droit civil.

114 ibid 3.2.2. en citant Göksu (n 41) n. 879; Gabrielle Kaufmann-Kohler et Antonio Rigozzi, Arbitrage international : droit et pratique à la lumière de la LDIP, $\left(2^{\mathrm{e}}\right.$, Bern 2010) 678. 
Dans le fameux arbitrage Yukos, la Russie a demandé au tribunal de district de La Haye d'annuler la sentence rendue par La Cour Permanente d'Arbitrage en 2014 ${ }^{115}$, entre autres motifs, parce que le tribunal arbitral n'avait pas rempli son mandat personnel ${ }^{116}$. Affirmant que Martin Valasek, qui avait été présenté comme assistant du président Fortier, avait joué un rôle important dans l'évaluation des preuves, les délibérations et la préparation des sentences finales, la Russie s'était référée à la feuille de présence du tribunal arbitral et du secrétaire pour révéler que M. Valasek avait consacré plus de temps à l'arbitrage qu'aucun des arbitres ${ }^{117}$. Le 20 avril 2016, le tribunal de district de La Haye a annulé six sentences arbitrales pour d'autres motifs, laissant la question sans discussion. ${ }^{118}$

L'une des déclarations les plus explicatives sur les fonctions des secrétaires d'arbitrage a peut-être été faite par Hon. M. le juge Popplewell dans l'affaire $P c$. $Q^{119}$. Dans cette affaire, un courrier électronique du président destiné au secrétaire avait été envoyé par erreur à un assistant juridique des avocats de P. Le courrier électronique contenait une lettre de $\mathrm{P}$ au tribunal et demandait sa « réaction à cette dernière de $[\mathrm{P}] »{ }^{120}$. P a contesté la sentence devant la Haute Cour d'Angleterre et du Pays de Galles, au motif notamment que le secrétaire était trop impliqué dans le processus de prise de décision. Dans la partie pertinente de la décision, le juge Popplewell a déclaré ce qui suit:

"Il y a une inquiétude considérable et compréhensible de la communauté des arbitres internationaux quant au fait que le recours aux secrétaires risque de les transformer en "quatrième arbitre". Il faut veiller à ce que la prise de décision soit bien celle des membres du tribunal. Le moyen le plus sûr de s'assurer que tel est le cas est que le secrétaire ne soit pas chargé de quoi que ce soit qui implique d'exprimer un point de vue sur le fond d'une demande ou d'une question. Si tel est le cas, le tribunal risque réellement de subir une influence inappropriée sur le processus décisionnel, ce qui compromettra sa capacité à parvenir à un jugement totalement indépendant. Le danger peut être plus grand avec des arbitres sans formation juridique ou sans expérience, qu'avec des juges qui sont habitués à prendre des décisions juridictionnelles entièrement indépendantes avec l'aide d'auxiliaires de justice ou autres assistants juridiques. Cependant, le danger existe pour tous les tribunaux. La meilleure pratique consiste donc à éviter d'impliquer un secrétaire de tribunal dans tout ce qui pourrait être qualifié d'expression d'un point de vue sur le fond de ce que le tribunal est appelé à trancher. Si le rôle du secrétaire est ainsi circonscrit, les parties peuvent être

115 Yukos Universal Limited (Isle of Man) v The Russian Federation, PCA Case No. AA 227, In the Matter of an Arbitration Before a Tribunal Constituted in Accordance with Article 26 of the Energy Charter Treaty and the 1976 UNCITRAL Arbitration Rules, Final Award, 18 juillet 2014. <https:/www.italaw.com/sites/default/files/case-documents/italaw3279. pdf> consulté le 10 septembre 2019.

116 Rechtbank Den Haag, ECLI:NL:RBDHA:2016:4230, C/09/477160 / HA ZA 15-1 Section 4.2. <http://uitspraken. rechtspraak.nl/inziendocument?id=ECLI:NL:RBDHA:2016:4230> consulté le 10 septembre 2019.

117 Writ of Summons (n 18) $185 \mathrm{ff}$.

118 Rechtbank Den Haag (n 116) Section 5.98.

119 [2017] EWHC 194 (Comm).

120 ibid [10]ff. 
assurées qu'il n'y a aucun risque d'influence inappropriée sur la fonction décisionnelle personnelle et non délégable du tribunal.

Toutefois, le fait de ne pas suivre les meilleures pratiques ne signifie pas ne pas mener correctement les procédures [...]. Pour l'arbitre, solliciter ou recevoir des avis de quelque nature que ce soit auprès du secrétaire d'un tribunal arbitral sur le fond des décisions ne prouve pas en soi qu'il manque à son devoir personnel de s'acquitter lui-même de la tâche décisive qui lui incombe. Cela est particulièrement vrai lorsque, comme en l'espèce, l'arbitre compétent est un juge expérimenté, habitué à prendre des décisions en toute indépendance et qui n'est pas influencé de manière inappropriée par les suggestions des assistants juridiques débutants. $»^{121}$

Justice Popplewell explique ensuite les missions réalisée par le secrétaire et démontre qu'il n'y a eu aucune faille quant au parfait déroulement de la procédure.

«Le recours à un secrétaire pour analyser les demandes et les projets d'ordonnances procédurales ne constitue pas une délégation indue de fonctions décisionnelles, sauf accord contraire des parties. Ce ne serait pas non plus nécessairement une délégation aussi inappropriée du président que de solliciter ou de prendre en compte l'opinion de ce secrétaire d'arbitrage sur le bien-fondé de ces décisions de procédure. [...] En tout état de cause, si le président avait demandé au secrétaire de donner son avis sur le bien-fondé de la demande, on ne pouvait en déduire (et le président a nié) qu'il avait ainsi abandonné une partie de son propre rôle de décision. $\gg^{122}$

Bien que les tribunaux nationaux semblent avoir été libéraux en ce qui concerne l'ampleur des tâches pouvant être confiées aux secrétaires, une contestation de la partie perdante concernant la délégation indue du mandat de l'arbitre peut entraîner une prolongation non désirée du litige.

\section{E. Minimiser les risques}

En citant le soutien apporté à la pratique consistant à utiliser des secrétaires au sein de la communauté arbitrale, Partasides et autres diraient que la plupart d'entre eux semblent accepter que ces défauts soient contrebalancés par les avantages inhérents à l'utilisation d'un secrétaire. ${ }^{123}$ Cependant, les risques peuvent aussi être minimisés.

L'inquiétude suscitée par la confidentialité, l'indépendance et l'impartialité des secrétaires semble être relativement facile à surmonter. Si un arbitre informe les parties de l'identité d'un secrétaire potentiel et ne le nomme pas sans le consentement des deux (ou de toutes les) parties, les parties peuvent déjà avoir une idée de l'indépendance et de l'impartialité de cette personne. Certaines règles institutionnelles, telles que la Note de la $\mathrm{CCI}^{124}$ et les guidelines de l'ACICA (Centre

121 ibid [68]-[69].

122 ibid [70].

123 Partasides et autres (n 10) 27.

124 Cour International d'Arbitrage (n 72) paras 2, 181 et 182. 
Australien d'Arbitrage Commercial International) ${ }^{125}$, prohibent déjà pour les arbitres de nommer un secrétaire sans en divulguer le nom et sans obtenir le consentement des parties. En outre, dans la convention d'arbitrage ou ultérieurement, les parties peuvent convenir de demander à un secrétaire potentiel de signer une convention de confidentialité ${ }^{126}$ et un engagement d'indépendance et d'impartialité. ${ }^{127}$

En ce qui concerne l'étendue des activités des secrétaires, dans les procédures ad hoc et même dans les cas où les parties choisissent une institution d'arbitrage dont les notes ou les directives ne sont pas contraignantes (ou bien lorsque l'institution arbitrale choisie n'a pas adopté de règlement concernant les secrétaires), les incertitudes peuvent être minimisées grâce à la transparence et au consentement éclairé des parties. ${ }^{128}$ Si un tribunal arbitral décrit clairement les tâches qu'un secrétaire sera en mesure d'accomplir ${ }^{129}$, obtient le consentement des parties et bénéficie de l'assistance de ce secrétaire de manière transparente et conformément à cette description, la question ne devrait pas susciter beaucoup d'inquiétude. Il est également possible pour les parties de s'entendre à l'avance sur les tâches du secrétaire à condition que le tribunal est transparent quant aux fonctions du secrétaire du début à la fin de la procédure. Après tout, un arbitre consciencieux et désireux de remplir son mandat avec diligence ne devrait avoir beaucoup de difficulté à appliquer la distinction claire qui existe entre délégation appropriée et dérogation irresponsable. ${ }^{130}$

Comme le dit Partasides, "The system should not be fashioned by fear of the irresponsible, for they can undermine any safeguard $» .{ }^{131}$ Compte tenu de tous les avantages qu'un secrétaire peut offrir et de tous les arbitres désireux de s'acquitter de leurs tâches de la manière la plus responsable, le recours à des secrétaires ne devrait pas être une pratique à éviter. Peut-être que dans quelques années, ce ne sera pas du « quatrième » mais du « cinquième arbitre $»^{132}$ dont il faudra être prudent. ${ }^{133}$

125 Australian Centre for International Commercial Arbitration (n 102) paras 1, 3 et 5.

126 Les guidelines de l'ACICA indiquent déjà qu' « [un] secrétaire de tribunal est soumis au même degré de confidentialité que celui requis des parties et du tribunal » ibid para 13

127 La note de la CPI exige également une déclaration d'indépendance et d'impartialité de la part du secrétaire potentiel. Cour International d'Arbitrage (n 72) para 181.

128 International Commercial Disputes Committee and Committee on Arbitration of the New York City Bar Association (n 4) 591; Douglas (n 12) 88; Maynard (n 45) 183; Conseil International pour l'Arbitrage Commercial (n 22) 16.

129 Pour un « Model Letter from Arbitral Tribunal to the Parties on the Appointment of an Arbitral Secretary or Assistant » voir, Kaufmann-Kohler et Rigozzi (n 5) 312.

130 Maynard (n 45) 182; Voir, Menz 2013 (n 52).

131 Partasides (n 7) 157.

132 Filipe Sanches Afonso, The Fifth Arbitrator? The Role of Artificial Intelligence to Tribunals in International Arbitration (Lisbon Arbitration 18 décembre 2018) <https://lisbonarbitration.mlgts.pt/articles/the-fifth-arbitrator-the-role-of-artificialintelligence-to-tribunals-in-international-arbitration/81/> consulté le 10 septembre 2019.

133 « Les arbitres doivent s'acquitter de leur mandat personnellement, sans déléger à un tiers. On peut se demander si l'utilisation par un tribunal d'une AI constitue une délégation acceptable de ses fonctions. " Maud Piers et Christian Aschauer, 'Conclusion' dans Maud Piers et Christian Aschauer (eds) Arbitration in the Digital Age The Brave New World of Arbitration 283, 294. 


\section{Conclusion}

Bien que les arbitres doivent s'acquitter de leurs obligations juridictionnelles sans délégation à un tiers en raison de la nature «intuitu personae » de leur mandat, cela ne signifie pas que les arbitres ne peuvent être assistés. Dans les grands arbitrages internationaux en particulier, le volume des documents soumis par les parties ainsi que la nécessité de prendre certaines dispositions peuvent laisser aux tribunaux une charge de travail accablante.

Le secrétaire du tribunal arbitral est une personne, généralement un jeune avocat travaillant au sein du cabinet d'avocats du président ou de l'arbitre unique, qui assiste le tribunal dans les tâches spécifiées par les arbitres. La question du secrétaire n'est généralement pas réglementé par la loi et, dans certaines juridictions, des incertitudes demeurent quant à la question de savoir si un tribunal arbitral peut nommer un secrétaire d'arbitrage sans l'approbation des parties.

Les secrétaires d'arbitrage peuvent améliorer l'efficacité du tribunal en laissant aux arbitres plus de temps pour se concentrer sur les questions de fond en cause et peuvent aussi réduire les frais généraux du tribunal arbitral, en particulier dans les cas où les arbitres sont rémunérés à l'heure. De plus, les jeunes collaborateurs peuvent bénéficier de la possibilité d'observer le déroulement d'un arbitrage du point de vue du secrétariat.

En revanche, l'utilisation de secrétaires d'arbitrage peut comporter des risques liés à l'indépendance et l'impartialité ainsi qu'à la confidentialité. C'est particulièrement le cas dans les hypothèses où des secrétaires sont nommés sans aucune information des parties. Par ailleurs, beaucoup d'auteurs ont exprimé leur inquiétude de voir les secrétaires devenir des décideurs. Quant à la question de savoir quelles tâches enfreignent la mission intuitu personae des arbitres, on peut trouver différentes réponses dans le milieu ; les points de vue des commentateurs, les règlements des institutions d'arbitrage et les décisions de justice.

Si les arbitres demandent l'approbation des parties pour la nomination des secrétaires, les risques peuvent être minimisés en demandant au secrétaire potentiel de signer un accord de confidentialité et une déclaration d'indépendance et d'impartialité. En ce qui concerne l'influence non-désirée que pourrait avoir un secrétaire sur la décision du tribunal, et si les parties ne s'étaient pas entendues à l'avance, les risques peuvent être minimisés par un arbitre consciencieux décrivant clairement les tâches qu'un secrétaire serait en mesure d'accomplir, obtenant le consentement des parties et déléguant les tâches avec diligence, transparence et conformément à la description.

Grant Support: The author received no grant support for this work. 


\section{Bibliography}

Afonso FS, The Fifth Arbitrator? The Role of Artificial Intelligence to Tribunals in International Arbitration (Lisbon Arbitration 18 décembre 2018) <https://lisbonarbitration.mlgts.pt/ articles/the-fifth-arbitrator-the-role-of-artificial-intelligence-to-tribunals-in-internationalarbitration/81/> consulté le 10 septembre 2019.

Aguilar-Alvarez G, 'Foreword' dans Conseil International pour l'Arbitrage Commercial, Young ICCA Guide on Arbitral Secretaries (The ICCA Reports No.1) (International Council for Commercial Arbitration 2014) vii

Akıncı Z, Milletlerarası Tahkim (4e, Vedat Kitapçılık 2016).

Alangoya YH, Medeni Usul Hukukumuzda Tahkimin Niteliği ve Denetlenmesi (İstanbul Üniversitesi 1973).

Andersson S, 'A Fourth Arbitrator or an Administrative Secretary? A Study on the Appointment and Authority of Arbitral Secretaries in Swedish Arbitral Proceedings' (Mémoire de maîtrise en droit de l'arbitrage, Université d'Uppsala, 2015).

Ashford P, Handbook on International Commercial Arbitration (Juris 2009).

Australian Centre for International Commercial Arbitration, ACICA Guideline on the Use of Tribunal Secretaries <https://acica.org.au/wp-content/uploads/2017/01/ACICA-TribunalSecretary-Guideline.pdf> consulté le 10 septembre 2019.

Berger B et Kellerhals F, International and Domestic Arbitration in Switzerland (3 ${ }^{\mathrm{e}}$, Beck/Hart 2015)

Berwin Leighton Paisner, Research based report on the use of tribunal secretaries in international commercial arbitration (2015 Berwin Leighton Paisner) <https://www-staging.bclplaw.com/ images/content/1/4/v2/147226/BLP-International-Arbitration-Survey-2015.pdf $>$ consulté le 10 septembre 2019.

Blackaby $\mathrm{N}$ et autres, Redfern and Hunter on International Arbitration (6 ${ }^{\mathrm{e}}$, Oxford University Press 2015).

Blavi F et Vial G, 'The Tribunal Secretary in International Arbitrations' (2017) 30 New York International Law Review 1.

Boltenko O, 'Stop Ignoring the Elephant in the Room!' (Kluwer Arbitration Blog 9 juin 2014) $<$ arbitrationblog.kluwerarbitration.com/2014/06/09/stop-ignoring-the-elephant-in-theroom-2/> consulté le 10 septembre 2019.

Born G, International Commercial Arbitration (2 $2^{\mathrm{e}}$, Kluwer Law International 2014).

Caner BK, 'Enerji Şartı Anlaşmasının Muvakkaten Uygulanması ve Anlaşmadan Çekilme Hakkında Bir Değerlendirme' (2014)3 Ankara Barosu Dergisi 315.

Cavalieros P, 'La Confidentialité de l'Arbitrage' (2006)2 Les Cahiers de l'Arbitrage 56.

Chambre de Commerce Internationale Cour International d'Arbitrage, Note aux Parties et aux Tribunaux Arbitraux sur la Conduite de l'Arbitrage selon le Reglement d'Arbitrage CCI (Cour International d'Arbitrage 2019) <https://cms.iccwbo.org/content/uploads/sites/3/2016/11/iccnote-to-parties-and-arbitral-tribunals-on-the-conduct-of-arbitration-french.pdf $>$ consulté le 10 septembre 2019.

Clay T, L'arbitre (Dalloz 2001).

Commission des Nations Unies Pour le Droit Commercial International, Aide-mémoire de la CNUDCI sur l'organisation des procédures arbitrales (Mars 2012)<https://www.uncitral.org/pdf/ french/texts/arbitration/arb-notes/arb-notes-2016-ebook-f.pdf> consulté le 10 septembre 2019. 
Commission des Nations Unies Pour le Droit Commercial International 'Draft Notes on Organizing Arbitral Proceedings: report of the Secretary-General (A/CN.9/423)' dans Yearbook of the United Nations Commission on International Trade Law Vol. XXVII (A/CN.9/SER.AI) (United Nations 1996) 45.

Conseil International pour 1'Arbitrage Commercial, Young ICCA Guide on Arbitral Secretaries (The ICCA Reports No.1) (International Council for Commercial Arbitration 2014).

Dayındarlı K, 'İhtiyarî Tahkimde Hakem Ücretleri' dans Karayalçın Y (ed), Prof. Dr. Ali Bozer'e Armağan, (Bankacılık Enstitüsü Yayınları 1998) 539.

Douglas Z, 'The Secretary to the Arbitral Tribunal' dans Bernhard Berger et Michael E. Schneider (eds) Inside the Black Box: How Arbitral Tribunals Operate and Reach Their Decisions (ASA Special Series No. 42) (Juris 2014) 87.

Eisemann F, 'Déontologie de L'Arbitre Commercial International' (1969) 4 Revue de I'Arbitrage 217.

Feit M et Chassot CT, 'The Swiss Federal Supreme Court Provides Guidance on the Proper Use of Arbitral Secretaries and Arbitrator Consultants under the Swiss lex arbitri: Case Note on DFC 4A_709/2014 dated 21 May 2015' (2015) 33 ASA Bulletin 897, 907.

Finland Arbitration Institute, Note on the Use of a Secretary <https://arbitration.fi/wp-content/ uploads/sites/22/2013/06/note-on-the-use-of-a-secretary.pdf> consulté le 10 septembre 2019.

Gaillard E et Savage J (eds), Fouchard, Gaillard, Goldman on International Commercial Arbitration (Kluwer Law International 1999).

Göksu T, Schiedsgerichtsbarkeit (Dike 2014).

Gómez KF, Key Duties of International Investment Arbitrators: A Transnational Study of Legal and Ethical Dilemmas (Springer 2019).

Heuman L, Arbitration Law of Sweden: Practice and Procedure (Juris 2003).

Hong Kong International Arbitration Center, Guidelines on the Use of a Secretary to the Arbitral Tribunal <https://www.hkiac.org/images/stories/arbitration/HKIAC\%20Guidelines\%20on\%20 Use $\% 20$ of $\% 20$ Secretary\%20to\%20Arbitral\%20Tribunal\%20-\%20Final.pdf> consulté le 10 septembre 2019.

Hughes B, 'The Problem of Undisclosed Assistance to Arbitral Tribunals' dans Patricia Shaughnessy et Sherling Tung, The Powers and Duties of an Arbitator: Liber Amicorum Pierre A. Karrer (Kluwer Law International 2017) 161.

İnci B, 'Hakem Sözleşmesi ve Hakem Yargılaması' (2006) 67 Türkiye Barolar Birliği Dergisi 380. Jensen Ole, Tribunal Secretaries in International Arbitration (Oxford University Press 2019).

Joint Report of the International Commercial Disputes Committee and the Committee on Arbitration of the New York City Bar Association, Secretaries to International Arbitral Tribunals, (2006) 17 American Review of International Arbitration 575.

Jones D, 'Ethical Implications of Using Paralegals and Tribunal Secretaries' (2014) 17 Hors Serie 251.

Judicial Arbitration and Mediation Services, Guidelines for Use of Clerks and Tribunal Secretaries in Arbitrations <https://www.jamsadr.com/files/Uploads/Documents/JAMS-InternationalGuidelines-for-Use-of-Clerks-and-Tribunal-Secretaries-in-Arbitrations.pdf $>$ consulté le 10 septembre 2019.

Karadelis K, 'The Role of the Tribunal Secretary' (Global Arbitration Review 21 décembre 2011) $<$ www.globalarbitrationreview.com/news/article/30051/the-role-tribunal-secretary/> consulté le 10 septembre 2019. 
Kaufmann-Kohler G et Rigozzi A, Arbitrage international : droit et pratique à la lumière de la LDIP, (2 ${ }^{\mathrm{e}}$, Bern 2010).

Kaufmann-Kohler G et Rigozzi A, International Arbitration: Law and Practice in Switzerland (3ème, Oxford University Press 2015).

Kayalı D, Milletlerarası Ticarî Tahkimde Hakemlerin Bağımsızlığı ve Tarafsızlığı (Seçkin 2015).

Keutgen G et Dal G (avec la collaboration de Dal M et Matray G), L'arbitrage en droit belge et international (3 $3^{\mathrm{e}}$, Bruylant 2015).

Kleiman E, 'Arbitre, Intuitu Personae' dans Laurent Lévy et Yves Derains (eds) Liber Amicorum en l'honneur de Serge Lazareff (Pedone 2011) 361.

Lalive P, 'Inquiétantes dérives de l'arbitrage CCI (sur un recent "Oukase” du Secrétariat de la Cour d'Arbitrage CCI)' (1995) ASA Bulletin 634.

Lalive P, 'L'Article 190 al. 2 LDIP a-t-il une utilité ?' (2010) 28 ASA Bulletin, 726.

Lalive P, 'Le choix de l'arbitre' dans Mélanges Jacques Robert, Libertés, (Montchrestien 1998) 353.

Lalive P, 'Mission et Démission des Arbitres Internationaux' dans Marcelo Kohen, Robert Kolb, Djacoba Liva Tehindrazanarivelo (eds) Perspectives of International Law in the 21st Century / Perspectives du Droit International au 21e Siecle: Liber Amicorum Professor Christian Dominica in Honour of His 80th Birthday (Bilinguale, Brill-Nijhoff 2011) 269.

Lalive P, 'Un Post-Scriptum et Quelques Citations' (1996) ASA Bulletin 35.

London Court of International Arbitration, LCIA Notes for Arbitrators, <https://www.lcia.org/adrservices/lcia-notes-for-arbitrators.aspx > consulté le 10 septembre 2019.

Loquin E, 'Les obligations de confidentialité dans l'arbitrage' (2006)2 Revue de l'Arbitrage 323.

Malacka M, 'Party Autonomy in the Procedure of Appointing Arbitrators' (2017) 17(2) International and Comparative Law Review 93.

Marguerat J et Blakemore TN, 'Note: A. SA v. B. Sàrl, Federal Supreme Court of Switzerland, 1st Civil Law Chamber, Case No. 4A_709/2014, 21 May 2015’ 201613 Revista Brasileira de Arbitragem 199.

Maynard S, 'Laying the fourth arbitrator to rest: re-evaluating the regulation of arbitral secretaries' (2018) 34 Arbitration International 173.

Mcasey B, 'International Arbitral Institutions' dans Freya Baetens (ed), Legitimacy of Unseen Actors in International Adjudication (Cambridge University Press 2019) 51.

Menz JU et George A, 'How Much Assistance Is Permissible? A Note on the Swiss Supreme Court's Decision on Arbitral Secretaries and Consultants' (2016) 33 Journal of International Arbitration 311, 313.

Menz JU, Miss Moneypenny vs. the Fourth Musketeer: the Role of Arbitral Secretaries (Kluwer Arbitration Blog 9 juillet 2013) < arbitrationblog.kluwerarbitration.com/2013/07/09/missmoneypenny-vs-the-fourth-musketeer-the-role-of-arbitral-secretaries/> consulté le 10 septembre 2019.

Mistelis LA, 'Efficiency. What Else? Efficiency as the Emerging Defining Value of International Arbitration: between Systems theories and party autonomy' (15 avril 2019) Queen Mary School of Law Legal Studies Research Paper No. 313/2019 <https://ssrn.com/abstract=3372341> consulté le 10 septembre 2019. 
Moses ML, The Principles and Practice of International Commercial Arbitration (Cambridge University Press 2018).

Mosk RM, 'Deliberations of Arbitrators' dans Caron D et autres (eds), Practising Virtue: Inside International Arbitration 486.

Özdem BH et Sert MY, 'Uluslararası Tahkimde Çıkar Çatışması Hakkında Uluslararası Barolar Birliği Kılavuzu'nun İncelenmesi' (2019) 8(1) Uluslararası Ticaret ve Tahkim Hukuku Dergisi 151.

Özsunay E, ‘Tahkim Yargılamasında Mahremiyet' (2004)2 İstanbul Barosu Dergisi 89.

Partasides C et autres, 'Arbitral Secretaries' dans Conseil International pour l'Arbitrage Commercial, Young ICCA Guide on Arbitral Secretaries (The ICCA Reports No.1) (International Council for Commercial Arbitration 2014) 23.

Partasides C, 'The Fourth Arbitrator? The Role of Secretaries to Tribunals in International Arbitration' (2002) 18 Arbitration International 147.

Partida S, L'arbitre international : étude de droit comparé (Master 2 Recherche de Droit européen comparé, Université Paris II Panthéon - Assas, Séjour de recherche à la Harvard Law School 2011) < <https://docassas.u-paris2.fr/nuxeo/site/esupversions/6078e606-e9d8-4733-b52a1bdd14eb402f $>$ consulté le 10 septembre 2019.

Piers M et Aschauer C, 'Conclusion' dans Piers M et Aschauer C (eds) Arbitration in the Digital Age The Brave New World of Arbitration 283.

Polkinghorne $\mathrm{M}$ et Rosenberg C, 'The Role of the Tribunal Secretary in International Arbitration: A Call for a Uniform Standard' (2014) 8 Dispute Resolution International 107.

Poudret J et Besson S, Comparative Law of International Arbitration (Sweet \& Maxwell 2007).

Redfern A et autres, Law and Practice of International Arbitration (4 $4^{\mathrm{e}}$, Sweet \& Maxwell 1999).

Restemayer CJ, 'Secretaries Always Get a Bad Rep: Identifying the Controversy Surrounding Administrative Secretaries, Current Guidelines, and Recommendations' (2012) 4 Yearbook on Arbitration Mediation 328.

Reyhani Yüksel S, 'Tahkim Tarafları ve Hakem Arasında Yapılan Hakemlik Sözleşmesinin Maddi Hukuk Açısından Değerlendirilmesi' (2016) 22 Marmara Üniversitesi Hukuk Fakültesi Hukuk Araştırmaları Dergisi: Özel Sayı Prof. Dr. Cevdet Yavuz’a Armağan 2436.

Rovine AW, Contemporary Issues in International Arbitration and Mediation: The Fordham Papers (Brill - Nijhoff 2010).

Rubino-Sammartano M, International Arbitration Law and Practice, (3 $3^{\mathrm{e}}$, Juris 2014).

Schwartz EA, 'The Rights and Duties of ICC Arbitrators' dans Jean-François Bourque (ed) Special Status of the Arbitrator, ICC International Court of Arbitration Bulletin: 1995 Special Supplement (ICC Publishing 1995) 67.

Schweizer P, 'Correspondance Au Sujet de L'Article 190(2) LDP: Quelques lignes en réponse à l'article du Professeur Lalive « L'article 190 al. 2 LDIP a-t-il une utilité ? »' (2011) 29 ASA Bulletin 66.

Singapore International Arbitration Center, Practice Note for Administered Cases - On the Appointment of Administrative Secretaries (2 février 2015) <http://www.siac.org.sg/our-rules/ practice-notes/practice-note-for-administered-cases-on-the-appointment-of-administrativesecretaries> consulté le 10 septembre 2019. 
SOAS University of London et Broderick Bozimo Company, Enquête SOAS sur l'Arbitrage en Afrique Arbitrage Interne et International: Perspectives par les praticiens africains de l'arbitrage $2018<$ https://eprints.soas.ac.uk/26110/1/Enquête\%20SOAS\%20sur\%201\%27arbitrage\%20 en\%20Afrique-Fr.pdf $>$ consulté le 10 septembre 2019.

Souleye A, 'Fourth chair: the controversial role of arbitral tribunal secretaries' (Young ICCA Blog 16 Février 2017) <http://www.youngicca-blog.com/fourth-chair-the-controversial-role-ofarbitral-tribunal-secretaries/> consulté le 10 septembre 2019.

Taşkın A, Hakem Sözleşmesi, (Turhan Kitabevi 2005).

Tarman ZD, ‘Tahkim ve Gizlilik’ (2008)1 Maltepe Üniversitesi Hukuk Fakültesi Dergisi 305.

Timlin T, 'The Swiss Supreme Court on the Use of Secretaries and Consultants in the Arbitral Process' (2016) 8 Yearbook on Arbitration Mediation 268.

Tüysüz C, 'Milletlerarası Ticari Tahkimde Gizlilik Yükümlülüğü' (2017) 37(2) Public and Private International Law Bulletin 846.

Üral N, 'Avrupa Enerji Şartı Antlaşmasının Onaylanmamış Olmasının Hakem Mahkemesinin Yetkisi Üzerindeki Etkileri: Yukos Davası' (2009) 8(1) İstanbul Kültür Üniversitesi Hukuk Fakültesi Dergisi 71.

Waincymer J, Procedure and Evidence in International Arbitration (Kluwer Law International 2012).

White \& Case et Queen Mary University of London School of International Arbitration, 2012 International Arbitration Survey: Current and Preferred Practices in the Arbitral Process, $<$ http://www.arbitration.qmul.ac.uk/media/arbitration/docs/2012_International_Arbitration_ Survey.pdf> consulté le 10 septembre 2019.

Yasan C, 'Milletlerarası Tahkimde Gizlilik' (2011)1 Galatasaray Üniversitesi Hukuk Fakültesi Dergisi 777.

Yıldırım F, 'Tahkimde İptal Davası ve İptal Davasının Amaçları Bakımından Bazı Değerlendirmeler' (2016) 22 Marmara Üniversitesi Hukuk Fakültesi Hukuk Araştırmaları Dergisi: Özel Sayı Prof. Dr. Cevdet Yavuz'a Armağan 3006.

Yu H et Ahmed M, 'Keeping the Invisible Hand under Control? -Arbitrator's Mandate and Assisting Third Parties' (2016) 19(2) Vindobona Journal of International Commercial Law and Arbitration 213. 
